\title{
Normal semicontinuity and the Dedekind completion of pointfree function rings
}

\author{
Javier Gutiérrez García, imanol Mozo Carollo, \\ AND JORGE PICADO
}

\begin{abstract}
This paper supplements an earlier one by the authors which constructed the Dedekind completion of the ring of continuous real functions on an arbitrary frame $L$ in terms of partial continuous real functions on $L$. In the present paper we provide three alternative views of it, in terms of (i) normal semicontinuous real functions on $L$, (ii) the Booleanization of $L$ (in the case of bounded real functions) and the Gleason cover of $L$ (in the general case) and (iii) Hausdorff continuous partial real functions on $L$. The first is the normal completion and extends Dilworth's classical construction to the pointfree setting. The second shows that in the bounded case the Dedekind completion is isomorphic to the lattice of bounded continuous real functions on the Booleanization of $L$ and that in the non-bounded case it is isomorphic to the lattice of continuous real functions on the Gleason cover of $L$. Finally, the third is the pointfree version of Anguelov's approach in terms of interval-valued functions. Two new classes of frames, cb-frames and weak cb-frames, emerge naturally in the first two representations. We show that they are conservative generalizations of their classical counterparts.
\end{abstract}

\section{Introduction}

Let $L$ be a frame and let $\mathrm{C}(L)$ (resp. $\mathrm{C}^{*}(L)$ ) denote the lattice-ordered ring of continuous (resp. bounded continuous) real functions on $L$. It is well known that $\mathrm{C}(L)$ and $\mathrm{C}^{*}(L)$ are distributive lattices. In general, however, they are not Dedekind complete: arbitrary non-void sets of continuous real functions in $\mathrm{C}(L)$ and $\mathrm{C}^{*}(L)$ bounded from above need not have a least upper bound in the lattices $\mathrm{C}(L)$ and $\mathrm{C}^{*}(L)$.

In a recent paper [29], we have constructed the Dedekind order completions $\mathrm{C}(L)^{\text {W }}$ and $\mathrm{C}^{*}(L)^{\text {x }}$ of respectively $\mathrm{C}(L)$ and $\mathrm{C}^{*}(L)$ in terms of the frame of partially defined real numbers and the corresponding classes of continuous partial real functions on the given frame $L$. In the present paper, we establish an alternative construction of the completion by means of normal subsets of $\mathrm{C}(L)$; we use for this purpose the ring $\mathrm{F}(L)$ of all real functions on $L$ (see [18]) and a special class of lower semicontinuous real functions, called normal [21],

2010 Mathematics Subject Classification: Primary: 06D22; Secondary: 06F25, 13J25, 26A15, 54C30, 54D15.

Key words and phrases: frame, locale, sublocale lattice, frame of (extended) reals, (extended) real function, continuous real function, function ring, Dedekind completion, cbframe, normal semicontinuous real function, Booleanization, Gleason cover, partial reals, partial real function, Hausdorff continuous real function. 
which are characterized by the property

$$
f^{-} \in \mathrm{F}(L) \quad \text { and } \quad\left(f^{-}\right)^{\circ}=f,
$$

where $f^{\circ}$ and $f^{-}$denote the lower and upper regularizations of $f$, respectively. Specifically, it is proved that the completions of $\mathrm{C}(L)$ and $\mathrm{C}^{*}(L)$ by normal subsets are respectively isomorphic with the lattices

$$
\begin{aligned}
\mathrm{C}(L)^{\#}=\{f \in \mathrm{F}(L) \mid f & \text { is normal lower semicontinuous and } \\
& \text { there exist } g, h \in \mathrm{C}(L) \text { such that } g \leqslant f \leqslant h\}
\end{aligned}
$$

and

$$
\begin{aligned}
\mathrm{C}^{*}(L)^{\#} & =\{f \in \mathrm{F}(L) \mid f \text { is normal lower semicontinuous and } \\
& \text { there exist } \left.g, h \in \mathrm{C}^{*}(L) \text { such that } g \leqslant f \leqslant h\right\} \\
& =\left\{f \in \mathrm{F}^{*}(L) \mid f \text { is normal lower semicontinuous }\right\} .
\end{aligned}
$$

The reader certainly recognizes here the classical description of the completion of $\mathrm{C}(X)$ due to Dilworth [11, Theorem 4.1], and simplified by Horn [22, Theorem 11] using lower semicontinuous real functions, usually referred to as the normal completion (cf. [24, 28]). Indeed, our results extend Dilworth's construction to the pointfree setting. But the pointfree situation is not merely a mimic of the classical one; there are some differences making the whole picture much more interesting. To put this is perspective, consider a completely regular topological space $(X, \mathcal{O} X)$ and the classes

$$
\begin{aligned}
\mathrm{C}(X) & =\{f: X \rightarrow \mathbb{R} \mid f \text { is continuous }\}, \\
\mathrm{C}^{*}(X) & =\{f: X \rightarrow \mathbb{R} \mid f \text { is continuous and bounded }\}, \\
\overline{\mathrm{C}}(X) & =\{f: X \rightarrow \overline{\mathbb{R}} \mid f \text { is continuous }\}
\end{aligned}
$$

(where $\overline{\mathbb{R}}$ denotes the extended real line $\mathbb{R} \cup\{-\infty,+\infty\}$ ). It is well known that the following statements are equivalent $[33,30,14]$ :

(1) $\mathrm{C}(X)$ is Dedekind complete.

(2) $\mathrm{C}^{*}(X)$ is Dedekind complete.

(3) $\overline{\mathrm{C}}(X)$ is Dedekind complete.

(4) $X$ is extremally disconnected.

The case $\mathcal{O} X=\mathcal{P}(X)$ (i.e., the discrete topology) being trivially extremally disconnected yields the well-known fact that $\mathrm{F}(X), \mathrm{F}^{*}(X)$ and $\overline{\mathrm{F}}(X)$ are all Dedekind complete. This simple fact is used in the construction of the Dedekind completion of $\mathrm{C}(X)$ (cf. [22]). The idea is that since $\mathrm{C}(X)$ is included in $\mathrm{F}(X)$ and the latter is Dedekind complete, one may find the Dedekind completion of $\mathrm{C}(X)$ inside $\mathrm{F}(X)$.

In the pointfree setting, however, the situation is somewhat distinct because the frame of all sublocales of a frame $L$ is not necessarily extremally disconnected. This means that, contrarily to $\mathrm{F}(X), \mathrm{F}(L)$ is not necessarily complete (indeed, given a non-void $\mathcal{F} \subseteq \mathrm{F}(L)$ bounded above one cannot ensure the existence of the supremum $\bigvee \mathcal{F}$ in $\mathrm{F}(L)$, see the discussion in [20, Sections 3.2 
and 3.3]). Thus we cannot ensure a priori, as in spaces, that we can find the completion of $\mathrm{C}(L)$ inside $\mathrm{F}(L)$.

The representation result for the completion described above, in terms of normal semicontinuous real functions (studied in Section 4), is presented in Section 5. As an immediate consequence of it, we get that for a completely regular frame $L, \mathrm{C}(L)$ is Dedekind complete if and only if $L$ is extremally disconnected, a result originally due to Banaschewski and Hong [6].

Further, in Sections 6 and 7, we provide a second representation for the completion. In the bounded case (Section 6), it states that for any completely regular frame $L$, the normal completion of $\mathrm{C}^{*}(L)$ is isomorphic to the lattice of all bounded continuous real functions on another naturally determined frame. This is the pointfree counterpart of Dilworth [11, Theorem 6.1]. It states precisely the following: for any completely regular frame $L$, the normal completion of $\mathrm{C}^{*}(L)$ is isomorphic to $\mathrm{C}^{*}(\mathfrak{B}(L))$, where $\mathfrak{B}(L)$ denotes the Booleanization of $L$ [7]. In the general case $\mathrm{C}(L)$, treated in Section 7, the Gleason cover $\mathfrak{G}(L)$ [2] of $L$ takes the role of the Booleanization but an assumption on the frame $L$ is required, namely, that it is weakly continuously bounded. This is the pointfree counterpart of Mack-Johnson [28, Proposition 4.1]. It highlights a new class of frames introduced in the paper: the weakly continuously bounded frames. Continuously bounded frames are introduced and studied in Section 3, and their weak variant in Section 4.

Finally, "pour tripler notre délectation" [10], we present a third representation for the completion in terms of the so called Hausdorff continuous partial real functions providing the pointfree setting for Anguelov's approach [1] in terms of interval-valued functions (cf. [9]).

\section{Background}

For basic notations and facts about pointfree topology and lattice theory we refer to [26] and [31]. Below, we provide a brief survey of the background required for this paper.

2.1. Sublocales. A sublocale set (briefly, a sublocale) $S$ of a frame (= locale) $L$ is a subset $S \subseteq L$ such that

(S1) for every $A \subseteq S, \bigwedge A$ is in $S$, and

(S2) for every $s \in S$ and every $x \in L, x \rightarrow s$ is in $S$.

The system of all sublocales constitutes a co-frame with the order given by inclusion, meet coinciding with the intersection and the join given by $\bigvee S_{i}=$ $\left\{\bigwedge M \mid M \subseteq \bigcup S_{i}\right\}$; the top is $L$ and the bottom is the set $\{1\}$.

For notational reasons, we make the co-frame of all sublocales of a locale $L$ into a frame $\mathcal{S}(L)$ by considering the dual ordering: $S_{1} \leq S_{2}$ iff $S_{2} \subseteq S_{1}$. Thus, $\{1\}$ is the top and $L$ is the bottom in $\mathcal{S}(L)$ that we simply denote by 1 and 0 , respectively. 
For any $a \in L$, the sets $\mathfrak{c}(a)=\uparrow a$ and $\mathfrak{o}(a)=\{a \rightarrow b \mid b \in L\}$ are the closed and open sublocales of $L$, respectively. They are complements of each other in $\mathcal{S}(L)$. Furthermore, the map $a \mapsto \mathfrak{c}(a)$ is a frame embedding $L \hookrightarrow \mathcal{S}(L)$ providing an isomorphism $\mathfrak{c}$ between $L$ and the subframe $\mathfrak{c}(L)$ of $\mathcal{S}(L)$ consisting of all closed sublocales. On the other hand, denoting by $\mathfrak{o}(L)$ the subframe of $\mathcal{S}(L)$ generated by all $\mathfrak{o}(a)$, the correspondence $a \mapsto \mathfrak{o}(a)$ establishes a dual poset embedding $L \rightarrow \mathfrak{o}(L)$.

Given a sublocale $S$ of $L$, its closure and interior are defined by

$$
\bar{S}=\bigvee\{\mathfrak{c}(a) \mid \mathfrak{c}(a) \leqslant S\}=\mathfrak{c}(\bigwedge S) \quad \text { and } \quad S^{\circ}=\bigwedge\{\mathfrak{o}(a) \mid S \leqslant \mathfrak{o}(a)\} .
$$

They satisfy the following properties (where $S^{*}$ and $a^{*}$ denote the pseudocomplements of $S$ and $a$ respectively in $\mathcal{S}(L)$ and $L)$ :

(1) $\overline{1}=1, \bar{S} \leqslant S, \overline{\bar{S}}=\bar{S}$, and $\overline{S \wedge T}=\bar{S} \wedge \bar{T}$,

(2) $0^{\circ}=0, S^{\circ} \geq S, S^{\circ \circ}=S^{\circ}$, and $(S \vee T)^{\circ}=S^{\circ} \vee T^{\circ}$,

(3) $S^{\circ}=\left(\overline{S^{*}}\right)^{*}=\mathfrak{o}\left(\bigwedge S^{*}\right)$,

(4) $\mathfrak{c}(a)^{\circ}=\mathfrak{o}\left(a^{*}\right)$,

(5) $\overline{\mathfrak{o}(a)}=\mathfrak{c}\left(a^{*}\right)$.

A sublocale $S$ is said to be regular closed (resp. regular open) if $\overline{S^{\circ}}=S$ (resp. $\bar{S}^{\circ}=S$ ). It is not hard to see that $S$ is regular closed if and only if $S=\mathfrak{c}(a)$ for some regular element $a \in L$ (that is, such that $a^{* *}=a$ ), and dually that $S$ is regular open if and only if $S=\mathfrak{o}(a)$ for some regular $a$.

2.2. The frame of (extended) reals. There are various equivalent ways of introducing the frame of reals $\mathfrak{L}(\mathbb{R})[3]$. Here it will be useful to adopt the description used in [18] given by generators $(p,-)$ and $(-, p), p \in \mathbb{Q}$, and relations

(r1) $(p,-) \wedge(-, q)=0$ whenever $p \geq q$,

(r2) $(p,-) \vee(-, q)=1$ whenever $p<q$,

(r3) $(p,-)=\bigvee_{q>p}(q,-)$, for every $p \in \mathbb{Q}$,

(r4) $(-, p)=\bigvee_{q<p}(-, q)$, for every $p \in \mathbb{Q}$,

(r5) $\bigvee_{p \in \mathbb{Q}}(p,-)=1$

(r6) $\bigvee_{p \in \mathbb{Q}}(-, p)=1$.

The meet $(p,-) \wedge(-, q)$ is simply denoted by $(p, q)$.

By dropping relations $(\mathrm{r} 5)$ and $(\mathrm{r} 6)$ in the description of $\mathfrak{L}(\mathbb{R})$ above, we have the corresponding frame of extended reals $\mathfrak{L}(\overline{\mathbb{R}})[4]$.

Remark. The basic homomorphism $\varrho: \mathfrak{L}(\overline{\mathbb{R}}) \rightarrow \mathfrak{L}(\mathbb{R})$ factors as

$$
\mathfrak{L}(\overline{\mathbb{R}}) \stackrel{\nu_{\omega}}{\longrightarrow} \downarrow \omega \stackrel{k}{\longrightarrow} \mathfrak{L}(\mathbb{R}), \quad \omega=\bigvee\{(p, q) \mid p, q \in \mathbb{Q}\}
$$

where $\nu_{\omega}=(\cdot) \wedge \omega$ and $k$ is an isomorphism (it is obviously onto and has a right inverse by the very definition of $\mathfrak{L}(\mathbb{R}))$. 
2.3. (Extended) continuous real functions. For any frame $L$, a continuous real function [3] (resp. extended continuous real function [4]) on a frame $L$ is a frame homomorphism $f: \mathfrak{L}(\mathbb{R}) \rightarrow L$ (resp. $f: \mathfrak{L}(\overline{\mathbb{R}}) \rightarrow L$ ). We denote by $\mathrm{C}(L)$ (resp. $\overline{\mathrm{C}}(L))$ the collection of all (resp. extended) continuous real functions on $L$. The correspondences $L \mapsto \mathrm{C}(L)$ and $L \mapsto \overline{\mathrm{C}}(L)$ are functorial in the obvious way.

Remark. Using the basic homomorphism $\varrho: \mathfrak{L}(\overline{\mathbb{R}}) \rightarrow \mathfrak{L}(\mathbb{R})$ from Remark 2.2, the $f \in \mathrm{C}(L)$ are in a one-to-one correspondence with the $g \in \overline{\mathrm{C}}(L)$ such that $g(\omega)=1$ (just take $g=f \varrho$ ). In what follows we will keep the notation $\mathrm{C}(L)$ to denote also the class inside $\overline{\mathrm{C}}(L)$ of the $f$ 's such that $f(\omega)=1$.

$\mathrm{C}(L)$ and $\overline{\mathrm{C}}(L)$ are partially ordered by

$$
\begin{aligned}
f \leqslant g & \Longleftrightarrow f(p,-) \leqslant g(p,-) \quad \text { for all } p \in \mathbb{Q} \\
& \Longleftrightarrow g(-, q) \leqslant f(-, q) \quad \text { for all } q \in \mathbb{Q} .
\end{aligned}
$$

2.4. Arbitrary (extended) real functions. Notice that there is a bijection between the collection of all arbitrary real functions on a space $(X, \mathcal{O} X)$ and the collection of all continuous real functions on $(X, \mathfrak{P}(X))$. Now, for a general frame $L$, the role of the lattice $\mathfrak{P}(X)$ of all subspaces of $X$ should be taken by the frame $\mathcal{S}(L)$ of all sublocales of $L$. This justifies thinking of frame homomorphisms $\mathfrak{L}(\mathbb{R}) \rightarrow \mathcal{S}(L)$ as of arbitrary real functions on $L$. Consequently, an $f \in \mathrm{F}(L)=\mathrm{C}(\mathcal{S}(L))$ (resp. $f \in \overline{\mathrm{F}}(L)=\overline{\mathrm{C}}(\mathcal{S}(L))$ ) is called an arbitrary (resp. extended) real function on $L$.

Remark. By the isomorphism $\mathfrak{c}: L \simeq \mathfrak{c}(L)$, each $f \in \mathrm{C}(L)$ corresponds uniquely to an $g_{f} \in \mathrm{F}(L)$ (precisely the $g_{f}=\mathfrak{c} \cdot f$ ), and thus $\mathrm{C}(L)$ is equivalent to the set of all $g \in \mathrm{F}(L)$ such that $g(p,-)$ and $g(-, q)$ are closed for every $p, q \in \mathbb{Q}$. Throughout, we keep the notation $\mathrm{C}(L)$ to denote also this subclass of $\mathrm{F}(L)$. We proceed similarly with an $f \in \overline{\mathrm{C}}(L)$.

2.4.1. Semicontinuous real functions. An $f$ in $\mathrm{F}(L)$ or $\overline{\mathrm{F}}(L)$ is

(1) lower semicontinuous if $f(p,-) \in \mathfrak{c}(L)$ for every $p \in \mathbb{Q}$;

(2) upper semicontinuous if $f(-, q) \in \mathfrak{c}(L)$ for every $q \in \mathbb{Q}$.

We denote by

$$
\operatorname{LSC}(L), \quad \operatorname{USC}(L), \quad \overline{\operatorname{LSC}}(L) \quad \text { and } \quad \overline{\operatorname{USC}}(L)
$$

the classes of lower semicontinuous and upper semicontinuous members of $\mathrm{F}(L)$ and $\overline{\mathrm{F}}(L)$ respectively.

Remarks. (1) There is a dual order-isomorphism $-(\cdot): \overline{\operatorname{LSC}}(L) \rightarrow \overline{\mathrm{USC}}(L)$ defined by

$$
(-f)(-, r)=f(-r,-) \quad \text { for all } r \in \mathbb{Q} .
$$

When restricted to $\operatorname{LSC}(L)$ it becomes a dual isomorphism from $\operatorname{LSC}(L)$ onto $\operatorname{USC}(L)$. Its inverse, denoted by the same symbol, maps a $g \in \overline{\mathrm{USC}}(L)$ into $-g \in \overline{\mathrm{LSC}}(L)$ defined by $(-g)(r,-)=g(-,-r)$ for all $r \in \mathbb{Q}$. 
(2) Notice that $\mathrm{C}(L)=\operatorname{LSC}(L) \cap \operatorname{USC}(L)$ and $\overline{\mathrm{C}}(L)=\overline{\mathrm{LSC}}(L) \cap \overline{\mathrm{USC}}(L)$.

(3) Lower (resp. upper) semicontinuous mappings $\varphi: X \rightarrow \mathbb{R}$ are in a bijective correspondence with the members of $\operatorname{LSC}(\mathcal{O} X)(\operatorname{resp} . \operatorname{USC}(\mathcal{O} X))[19,20]$. Specifically, each lower semicontinuous $\varphi: X \rightarrow \mathbb{R}$ corresponds to the frame homomorphism $f_{\varphi}: \mathfrak{L}(\mathbb{R}) \rightarrow \mathcal{S}(\mathcal{O} X)$ given by

$$
f_{\varphi}(p,-)=\mathfrak{c}\left(\varphi^{-1}((p,+\infty))\right) \quad \text { and } \quad f_{\varphi}(-, q)=\bigvee_{s<q} \mathfrak{o}\left(\varphi^{-1}((s,+\infty))\right)
$$

for every $p, q \in \mathbb{Q}$, and, dually, each upper semicontinuous $\varphi: X \rightarrow \mathbb{R}$ corresponds to the upper semicontinuous real function $f_{\varphi}: \mathfrak{L}(\mathbb{R}) \rightarrow \mathcal{S}(\mathcal{O} X)$ given by

$$
f_{\varphi}(p,-)=\bigvee_{r>p} \mathfrak{o}\left(\varphi^{-1}((-\infty, r))\right) \quad \text { and } \quad f_{\varphi}(-, q)=\mathfrak{c}\left(\varphi^{-1}((-\infty, q))\right)
$$

for each $p, q \in \mathbb{Q}$. Their restrictions to continuous mappings $\varphi: X \rightarrow \mathbb{R}$ yield a bijection with the members of $\mathrm{C}(\mathcal{O} X)$, where the $f_{\varphi}$ is just given by

$$
f_{\varphi}(p,-)=\mathfrak{c}\left(\varphi^{-1}((p,+\infty))\right) \quad \text { and } \quad f_{\varphi}(-, q)=\mathfrak{c}\left(\varphi^{-1}((-\infty, q))\right) .
$$

Moreover, it is easy to check that these bijections are order preserving, i.e., given $\varphi_{1}, \varphi_{2}: X \rightarrow \mathbb{R}$, then $\varphi_{1} \leqslant \varphi_{2}$ if and only if $f_{\varphi_{1}} \leqslant f_{\varphi_{2}}$.

A similar situation holds in the case of extended real functions (see [4]).

2.5. Scales. There is a useful way of specifying (extended) continuous real functions on a frame $L$ with the help of the so called (extended) scales ([18, Section 4]). An extended scale in $L$ is a map $\sigma: \mathbb{Q} \rightarrow L$ such that $\sigma(p) \vee \sigma(q)^{*}=$ 1 whenever $p<q$. An extended scale is a scale if

$$
\bigvee_{p \in \mathbb{Q}} \sigma(p)=1=\bigvee_{p \in \mathbb{Q}} \sigma(p)^{*} .
$$

Remark. An (extended) scale is necessarily an antitone map. Conversely, if $\sigma$ is antitone and for each $p<q$ in $\mathbb{Q}$ there exists a complemented element $a_{p, q} \in L$ such that $\sigma(q) \leqslant a_{p, q} \leqslant \sigma(p)$, then $\sigma$ is an (extended) scale (indeed, $\sigma(p) \vee \sigma(q)^{*} \geq a_{p, q} \vee a_{p, q}{ }^{*}=1$ whenever $\left.p<q\right)$. In particular, if all $\sigma(r)$ are complemented, then $\sigma$ is an (extended) scale if and only if it is antitone.

For each extended scale $\sigma$ in $L$, the formulas

$$
f(p,-)=\bigvee_{r>p} \sigma(r) \text { and } f(-, q)=\bigvee_{r<q} \sigma(r)^{*}, \quad p, q \in \mathbb{Q},
$$

determine an $f \in \overline{\mathrm{C}}(L)$; then, $f \in \mathrm{C}(L)$ if and only if $\sigma$ is a scale. Moreover, given $f, f_{1}, f_{2} \in \overline{\mathrm{C}}(L)$ determined by extended scales $\sigma, \sigma_{1}$ and $\sigma_{2}$, respectively, we have:

(1) $f(p,-) \leqslant \sigma(p) \leqslant f(-, p)^{*}$ for every $p \in \mathbb{Q}$.

(2) $f_{1} \leqslant f_{2}$ if and only if $\sigma_{1}(p) \leqslant \sigma_{2}(q)$ for every $p>q$ in $\mathbb{Q}$. 
Examples. For each $r \in \mathbb{Q}$, the scale $\sigma_{r}$ given by $\sigma_{r}(p)=0$ if $p \geq r$ and $\sigma_{r}(p)=1$ if $p<r$, determines the constant function $\boldsymbol{r} \in \mathrm{C}^{*}(L)$, given by

$$
\boldsymbol{r}(p,-)=\left\{\begin{array}{ll}
0 & \text { if } p \geq r, \\
1 & \text { if } p<r,
\end{array} \text { and } \quad \boldsymbol{r}(-, p)= \begin{cases}1 & \text { if } p>r \\
0 & \text { if } p \leqslant r\end{cases}\right.
$$

One can similarly define two extended constant functions $+\infty$ and $-\infty$ generated by the extended scales $\sigma_{+\infty}: p \mapsto 1$ and $\sigma_{-\infty}: p \mapsto 0$. They are defined for each $p, q \in \mathbb{Q}$ by

$$
+\infty(p,-)=1=-\infty(-, q) \text { and } \quad+\infty(-, q)=0=-\infty(p,-),
$$

and they are precisely the top and bottom elements of $\overline{\mathrm{C}}(L)$.

Of course, we can also use scales in $\mathcal{S}(L)$ to determine arbitrary real functions on $L$.

2.6. Complete regularity. Given a frame $L$ and $a, b \in L, b$ is really inside $a$ (written: $b \prec \prec a$ ) if there exists a family $\left\{c_{r} \mid r \in \mathbb{Q} \cap[0,1]\right\} \subseteq L$ such that $b \leqslant c_{0}, c_{1} \leqslant a$ and $c_{r}^{*} \vee c_{s}=1$ whenever $r<s$. A frame $L$ is called completely regular if $a=\bigvee\{b \in L \mid b \prec \prec a\}$ for every $a \in L$. The following result was proved in [15]:

Proposition. Let $L$ be a frame and $a, b \in L$. Then

(1) $b \prec \prec a$ if and only if there exists an $f \in \mathrm{C}(L)$ satisfying $0 \leqslant f \leqslant \mathbf{1}$ such that $\mathfrak{c}(b) \leqslant f(-, 1)^{*}$ and $f(0,-) \leqslant \mathfrak{c}(a)$.

(2) $L$ is completely regular if and only if for each $S \in \mathfrak{c}(L)$,

$$
\begin{array}{r}
S=\bigvee\left\{T \in \mathfrak{c}(L) \mid \text { there exists } f_{T} \in \mathrm{C}(L) \text { satisfying } 0 \leqslant f_{T} \leqslant \mathbf{1},\right. \\
\left.\qquad \leqslant f_{T}(-, 1)^{*} \text { and } f_{T}(0,-) \leqslant S\right\} .
\end{array}
$$

\section{Bounded real functions and cb-frames}

Let us remind the reader that a real function $f \in \mathrm{F}(L)$ is bounded if there exist $p<q$ in $\mathbb{Q}$ such that $f(p,-)=1=f(-, q)$. Equivalently, this means that there exist $p<q$ in $\mathbb{Q}$ such that $\boldsymbol{p} \leqslant f \leqslant \boldsymbol{q}$ (i.e., $f(-, p)=0=f(q,-)$ ). In this section we will discuss some variants of boundedness for general real functions that will play an important role in our results.

Definition 3.1. We say that $f$ is

(1) continuously bounded if there exist $h_{1}, h_{2} \in \mathrm{C}(L)$ such that $h_{1} \leqslant f \leqslant h_{2}$;

(2) locally bounded if

$$
\bigvee_{r \in \mathbb{Q}} \overline{f(r,-)}=1=\bigvee_{r \in \mathbb{Q}} \overline{f(-, r)}
$$


We denote by $\mathrm{F}^{*}(L), \mathrm{F}^{c b}(L)$ and $\mathrm{F}^{l b}(L)$ the collections of all bounded, continuously bounded and locally bounded members of $\mathrm{F}(L)$ respectively. Similarly we have the classes

$$
\operatorname{LSC}^{*}(L), \operatorname{LSC}^{c b}(L), \operatorname{LSC}^{l b}(L), \operatorname{USC}^{*}(L), \operatorname{USC}^{c b}(L) \text { and } \operatorname{USC}^{l b}(L) .
$$

Remarks 3.2. (1) It readily follows from the definitions that

$$
\mathrm{F}^{*}(L) \subseteq \mathrm{F}^{c b}(L) \subseteq \mathrm{F}^{l b}(L) \subseteq \mathrm{F}(L) .
$$

(2) Note that $f \in \operatorname{LSC}^{l b}(L)$ if and only if $f \in \operatorname{LSC}(L)$ and $\bigvee_{r \in \mathbb{Q}} \overline{f(-, r)}=1$ and, dually, $f \in \operatorname{USC}^{l b}(L)$ if and only if $f \in \operatorname{USC}(L)$ and $\bigvee_{r \in \mathbb{Q}} \overline{f(r,-)}=1$.

(3) Recall that a real function $\varphi: X \rightarrow \mathbb{R}$ on a topological space $X$ is locally bounded if for every $x \in X$ there exists an open neighbourhood $U_{x}$ such that $\varphi\left(U_{x}\right)$ is bounded. Consequently, $\varphi$ is locally bounded if and only if

$$
\bigcup_{r \in \mathbb{Q}} \operatorname{Int}\left(\varphi^{-1}([r,+\infty))\right)=X=\bigcup_{r \in \mathbb{Q}} \operatorname{Int}\left(\varphi^{-1}((-\infty, r])\right),
$$

as can be easily checked. In particular, a lower semicontinuous $\varphi$ is locally bounded if and only if $\bigcup_{r \in \mathbb{Q}}$ Int $\left(\varphi^{-1}((-\infty, r))\right)=X$ and an upper semicontinuous $\varphi$ is locally bounded if and only if $\bigcup_{r \in \mathbb{Q}} \operatorname{Int}\left(\varphi^{-1}((r,+\infty))\right)=X$.

(4) Given a lower semicontinuous mapping $\varphi: X \rightarrow \mathbb{R}$ and the corresponding lower semicontinuous real function $f_{\varphi}$ in $\mathrm{F}(\mathcal{O} X)$ introduced in Remark 2.4.1(3) we have that:

(a) $\varphi$ is bounded if and only if $f_{\varphi}$ is bounded;

(b) $\varphi$ is continuously bounded if and only if $f_{\varphi}$ is continuously bounded;

(c) $\varphi$ is locally bounded if and only if $f_{\varphi}$ is locally bounded.

For the latter, we have the following proof: For any $\varphi \in \operatorname{LSC}(X)$, the condition of $\varphi$ being locally bounded means precisely that, in $\mathcal{S}(\mathcal{O} X)$,

$$
\begin{aligned}
1 & =\bigvee_{r \in \mathbb{Q}} \mathfrak{c}\left(\varphi^{-1}((r,+\infty))^{*}\right)=\bigvee_{r \in \mathbb{Q}} \overline{\mathfrak{o}\left(\varphi^{-1}((r,+\infty))\right)}, \quad \text { that is, } \\
1 & =\bigvee_{q \in \mathbb{Q}} \overline{\bigvee_{r<q} \mathfrak{o}\left(\varphi^{-1}((r,+\infty))\right)}=\bigvee_{q \in \mathbb{Q}} \overline{f_{\varphi}(-, q)}
\end{aligned}
$$

(notice that for each $r \in \mathbb{Q}$,

$$
\overline{\mathfrak{o}\left(\varphi^{-1}((r,+\infty))\right)} \leqslant \overline{\bigvee_{r<r+1} \mathfrak{o}\left(\varphi^{-1}((r,+\infty))\right)} \leqslant \bigvee_{q \in \mathbb{Q}} \overline{\bigvee_{r<q} \mathfrak{o}\left(\varphi^{-1}((r,+\infty))\right)},
$$

and

$$
\overline{\bigvee_{r<q} \mathfrak{o}\left(\varphi^{-1}((r,+\infty))\right)} \leqslant \overline{\mathfrak{o}\left(\varphi^{-1}((q,+\infty))\right)} \leqslant \bigvee_{r \in \mathbb{Q}} \overline{\mathfrak{o}\left(\varphi^{-1}((r,+\infty))\right)}
$$

for each $q \in \mathbb{Q})$. The last identity means that $f_{\varphi} \in \operatorname{LSC}^{l b}(\mathcal{O} X)$.

Dually, we have similar results for upper semicontinuous real functions.

The lower and upper regularizations of a real function on $L$ were introduced and studied in $[16,18]$. The lower regularization $f^{\circ}$ of an $f \in \overline{\mathrm{F}}(L)$ is the 
extended real function generated by the extended scale $\sigma_{f}: r \mapsto \overline{f(r,-)}$, i.e.,

$$
f^{\circ}(p,-)=\bigvee_{r>p} \overline{f(r,-)} \text { and } f^{\circ}(-, q)=\bigvee_{s<q}(\overline{f(s,-)})^{*} .
$$

Dually, the upper regularization $f^{-}$of $f$ is defined by $f^{-}=-(-f)^{\circ}$. Equivalently, $f^{-}$is the extended real function generated by the extended scale $\sigma_{f^{-}}: r \mapsto(\overline{f(-, r)})^{*}$, i.e.,

$$
f^{-}(p,-)=\bigvee_{r>p}(\overline{f(-, r)})^{*} \text { and } f^{-}(-, q)=\bigvee_{s<q} \overline{f(-, s)}
$$

The following basic properties (cf. $[16,18])$ of the operators

$$
(\cdot)^{\circ}: \overline{\mathrm{F}}(L) \rightarrow \overline{\mathrm{LSC}}(L) \text { and }(\cdot)^{-}: \overline{\mathrm{F}}(L) \rightarrow \overline{\mathrm{USC}}(L)
$$

will be useful in the sequel.

Proposition 3.3. [18, Propositions 7.3 and 7.4] The following hold for any $f, g \in \overline{\mathrm{F}}(L):$

(1) $(+\infty)^{\circ}=+\infty$ and $(-\infty)^{-}=-\infty$.

(2) $f^{\circ} \leqslant f \leqslant f^{-}$.

(3) $f^{\circ \circ}=f^{\circ}$ and $f^{--}=f^{-}$.

(4) $(f \wedge g)^{\circ}=f^{\circ} \wedge g^{\circ}$ and $(f \vee g)^{-}=f^{-} \vee g^{-}$. (Hence $f \leqslant g$ implies that $f^{\circ} \leqslant g^{\circ}$ and $\left.f^{-} \leqslant g^{-}.\right)$

(5) Both $(\cdot)^{\circ-}$ and $(\cdot)^{-\circ}$ are idempotent, i.e., $f^{\circ-\circ-}=f^{\circ-}$ and $f^{-\circ-\circ}=f^{-\circ}$.

As a corollary of Proposition 3.3 we have:

Corollary 3.4. Let $f \in \overline{\mathrm{F}}(L)$. Then:

(1) $\overline{\operatorname{LSC}}(L)=\left\{f \in \overline{\mathrm{F}}(L) \mid f=f^{\circ}\right\}, \overline{\mathrm{USC}}(L)=\left\{f \in \overline{\mathrm{F}}(L) \mid f^{-}=f\right\}$ and $\overline{\mathrm{C}}(L)=\left\{f \in \overline{\mathrm{F}}(L) \mid f^{\circ}=f=f^{-}\right\}$.

(2) $f^{\circ}=\bigvee\{g \in \overline{\operatorname{LSC}}(L) \mid g \leqslant f\}$ and $f^{-}=\bigwedge\{g \in \overline{\operatorname{USC}}(L) \mid g \geq f\}$.

In general, the regularization of a real function is an extended real function. However, we have the following:

Proposition 3.5 ([18, Proposition 7.8]). The following hold for any $f \in \mathrm{F}(L)$ :

(1) If $\bigvee_{p \in \mathbb{Q}} \overline{f(p,-)}=1$ then $f^{\circ} \in \mathrm{F}(L)$.

(2) If $\bigvee_{q \in \mathbb{Q}} \overline{f(-, q)}=1$ then $f^{-} \in \mathrm{F}(L)$.

Regarding locally bounded real functions, we have the following easy consequence:

Corollary 3.6. The following statements are equivalent for any $f \in \mathrm{F}(L)$ :

(1) $f$ is locally bounded.

(2) There exist $g \in \operatorname{LSC}(L)$ and $h \in \operatorname{USC}(L)$ such that $g \leqslant f \leqslant h$.

(3) $f^{\circ}, f^{-} \in \mathrm{F}(L)$.

(4) $f^{\circ}$ and $f^{-}$are locally bounded.

(5) $f^{\circ}, f^{-}, f^{\circ-}, f^{-\circ} \in \mathrm{F}(L)$.

(6) $f^{\circ}, f^{-}, f^{\circ-}$ and $f^{-\circ}$ are locally bounded. 
(7) $f^{\circ}, f^{-}, f^{\circ-}, f^{-\circ}, f^{\circ-\circ}, f^{-\circ-} \in \mathrm{F}(L)$.

(8) $f^{\circ}, f^{-}, f^{\circ-}, f^{-\circ}, f^{\circ-\circ}$ and $f^{-\circ-}$ are locally bounded.

Proof. (1) $\Longrightarrow(2)$ follows from Proposition 3.5 since $f^{\circ} \in \operatorname{LSC}(L), f^{-} \in$ $\mathrm{USC}(L)$ and $f^{\circ} \leqslant f \leqslant f^{-}$. (2) $\Longleftrightarrow(3)$ follows from Proposition 3.3. (4) $\Longrightarrow$ $(5) \Longrightarrow(6)$ and $(6) \Longrightarrow(7) \Longrightarrow(8)$ follow similarly as $(1) \Longrightarrow(3) \Longrightarrow(4)$.

$(3) \Longrightarrow(4)$ : Let $f \in \mathrm{F}(L)$ such that $f^{\circ}, f^{-} \in \mathrm{F}(L)$. By Proposition 3.3 (2) we know that $f^{\circ} \leq f^{-}$. Then one has

$$
\bigvee_{p \in \mathbb{Q}} \overline{f^{\circ}(-, p)} \geq \bigvee_{p \in \mathbb{Q}} \overline{f^{-}(-, p)}=\bigvee_{p \in \mathbb{Q}} f^{-}(-, p)=1
$$

and, similarly, one has

$$
\bigvee_{p \in \mathbb{Q}} \overline{f^{-}(p,-)} \geq \bigvee_{p \in \mathbb{Q}} \overline{f^{\circ}(p,-)}=\bigvee_{p \in \mathbb{Q}} f^{\circ}(p,-)=1
$$

By Remarks $3.2(2)$ we conclude that both $f^{\circ}$ and $f^{-}$belong to $\mathrm{F}^{l b}(L)$.

$(8) \Longrightarrow(1)$ : This is obvious since

$$
1=\bigvee_{p \in \mathbb{Q}} f^{\circ}(p,-)=\bigvee_{p \in \mathbb{Q}} \overline{f(p,-)} \quad \text { and } \quad 1=\bigvee_{q \in \mathbb{Q}} f^{-}(-, q)=\bigvee_{q \in \mathbb{Q}} \overline{f(-, q)}
$$

Definition 3.7. A frame $L$ is continuously bounded (shortly, a cb-frame) if every locally bounded real function on $L$ is bounded above by a continuous real function.

Proposition 3.8. The following are equivalent for a frame $L$ :

(1) L is continuously bounded.

(2) Every upper semicontinuous and locally bounded real function on $L$ is bounded above by a continuous real function.

(3) Every lower semicontinuous and locally bounded real function on $L$ is bounded below by a continuous real function.

(4) $\mathrm{F}^{c b}(L)=\mathrm{F}^{l b}(L)$.

Proof. (1) $\Longrightarrow(2)$ and (4) $\Longrightarrow(1)$ are obvious and $(2) \Longleftrightarrow(3)$ is also clear since $f \in \operatorname{LSC}(L)$ if and only if $-f \in \operatorname{USC}(L)$.

$(3) \Longrightarrow(4)$ : Let $f \in \mathrm{F}^{l b}(L)$. We can immediately derive from Corollary 3.6 that $f^{\circ},-f^{-} \in \operatorname{LSC}^{l b}(L)$. Our hypothesis implies that we may find $g_{1}, g_{2} \in$ $\mathrm{C}(L)$ such that $g_{1} \leqslant f^{\circ}$ and $g_{2} \leqslant-f^{-}$. Hence $g_{1} \leqslant f^{\circ} \leqslant f \leqslant f^{-} \leqslant-g_{2}$ and $f \in \mathrm{F}^{c b}(L)$.

Remark 3.9. Since the bijections in Remarks 2.4.1(3) and 3.2(4) are order preserving, it follows from Proposition 3.8 that continuous boundedness is a conservative extension of the classical notion (originally due to Horne [23], see also $[27,28])$, that is, a topological space $X$ is a cb-space if and only if $\mathcal{O} X$ is a cb-frame.

It also follows from the above result (using [17, Proposition 5.4]) that any normal and countable paracompact frame (in particular, any perfectly normal frame [17, Proposition 5.3]) is a cb-frame. 


\section{Normal semicontinuous real functions}

One can say more about $f^{\circ}$ and $f^{-}$in case $L$ is completely regular, as the following result shows. In its proof we use the formulas for the operations in the algebra $\mathrm{F}(L)$ obtained in [20] (cf. [3]).

Lemma 4.1. Let $L$ be a completely regular frame and $f \in \overline{\mathrm{F}}(L)$.

(1) If there exists $g_{0} \in \mathrm{C}(L)$ such that $g_{0} \leqslant f$, then

$$
f^{\circ}=\bigvee\{g \in \mathrm{C}(L) \mid g \leqslant f\}
$$

(2) If there exists $g_{0} \in \mathrm{C}(L)$ such that $f \leqslant g_{0}$, then

$$
f^{-}=\bigwedge\{g \in \mathrm{C}(L) \mid f \leqslant g\} .
$$

Proof. The proof follows the lines of [29, Lemma 3.1]. First note that by [20, Corollary 3.5],

$$
\bigvee\{g \in \mathrm{C}(L) \mid g \leqslant f\} \in \operatorname{LSC}(L) \quad \text { and } \quad \bigwedge\{g \in \mathrm{C}(L) \mid f \leqslant g\} \in \operatorname{USC}(L) \text {. }
$$

Then we only need to show that $f^{\circ} \leqslant \bigvee\{g \in \mathrm{C}(L) \mid g \leqslant f\}$ since the converse inequality is trivial and (2) follows easily from (1).

We fix $p \in \mathbb{Q}$ and consider $p^{\prime} \in \mathbb{Q}$ such that $p<p^{\prime}$. Since $L$ is completely regular, then by Proposition 2.6(2),

$$
\begin{array}{r}
\overline{f\left(p^{\prime},-\right)}=\bigvee\left\{S \in \mathfrak{c}(L) \mid \text { exists } h_{S} \in \mathrm{C}(L) \text { satisfying } \mathbf{0} \leqslant h_{S} \leqslant \mathbf{1},\right. \\
\left.S \leqslant h_{S}(-, 1)^{*} \text { and } h_{S}(0,-) \leqslant \overline{f\left(p^{\prime},-\right)}\right\} .
\end{array}
$$

Let $S \in \mathfrak{c}(L)$ be one of such closed sublocales and let

$$
g_{S}=g_{0}+\left(\left(\left(\boldsymbol{p}^{\prime}-g_{0}\right) \vee \mathbf{0}\right) \cdot h_{S}\right) \in \mathrm{C}(L) .
$$

We also have that $g_{S} \leqslant f$; indeed, for each $r \in \mathbb{Q}$,

$$
\begin{aligned}
& g_{S}(r,-)=\bigvee_{r^{\prime} \in \mathbb{Q}} g_{0}\left(r-r^{\prime},-\right) \wedge\left(\left(\left(\boldsymbol{p}^{\prime}-g_{0}\right) \vee \mathbf{0}\right) \cdot h_{S}\right)\left(r^{\prime},-\right) \\
& =\left(\underset{r^{\prime}<0}{\bigvee} g_{0}\left(r-r^{\prime},-\right)\right) \vee\left(\underset{r^{\prime} \geq 0}{\bigvee} g_{0}\left(r-r^{\prime},-\right) \wedge\left(\left(\left(\boldsymbol{p}^{\prime}-f\right) \vee \mathbf{0}\right) \cdot h_{S}\right)\left(r^{\prime},-\right)\right) \\
& =g_{0}(r,-) \vee\left(\underset{r^{\prime} \geq 0}{\bigvee} \underset{r^{\prime \prime}>0}{\bigvee} g_{0}\left(r-r^{\prime},-\right) \wedge\left(\left(\boldsymbol{p}^{\prime}-f\right) \vee \mathbf{0}\right)\left(r^{\prime \prime},-\right) \wedge h_{S}\left(\frac{r^{\prime}}{r^{\prime \prime}},-\right)\right) \\
& =g_{0}(r,-) \vee\left(\underset{r^{\prime} \geq 0}{\bigvee} \underset{r^{\prime \prime}>0}{\bigvee} g_{0}\left(r-r^{\prime}, p^{\prime}-r^{\prime \prime}\right) \wedge h_{S}\left(\frac{r^{\prime}}{r^{\prime \prime}},-\right)\right) \\
& =g_{0}(r,-) \vee\left(\bigvee_{r^{\prime} \geq 0} \underset{r^{\prime}<r^{\prime \prime}<p^{\prime}-r+r^{\prime}}{\bigvee} g_{0}\left(r-r^{\prime}, p^{\prime}-r^{\prime \prime}\right) \wedge h_{S}\left(\frac{r^{\prime}}{r^{\prime \prime}},-\right)\right) \text {. }
\end{aligned}
$$


Now, if $r \geq p^{\prime}$ then $p^{\prime}-r+r^{\prime} \leqslant r^{\prime}$ for each $r^{\prime} \geq 0$ and thus $g_{S}(r,-)=$ $g_{0}(r,-) \leqslant f(r,-)$. Otherwise, if $r<p^{\prime}$ then

$$
\begin{aligned}
g_{S}(r,-) & \leqslant g_{0}(r,-) \vee\left(\bigvee_{r^{\prime} \geq 0} r_{r^{\prime}<r^{\prime \prime}<p^{\prime}-r+r^{\prime}} g_{0}\left(r-r^{\prime}, p^{\prime}-r^{\prime \prime}\right) \wedge h_{S}(0,-)\right) \\
& =g_{0}(r,-) \vee\left(\bigvee_{r^{\prime} \geq 0} g_{0}\left(r-r^{\prime}, p^{\prime}-r^{\prime}\right) \wedge h_{S}(0,-)\right) \\
& =g_{0}(r,-) \vee\left(g_{0}\left(-, p^{\prime}\right) \wedge h_{S}(0,-)\right) \leqslant g_{0}(r,-) \vee h_{S}(0,-) \\
& \leqslant f(r,-) \vee \overline{f\left(p^{\prime},-\right)} \leqslant f(r,-) \vee f\left(p^{\prime},-\right)=f(r,-) .
\end{aligned}
$$

Therefore $g_{S}(r,-) \leqslant f(r,-)$ for every $r \in \mathbb{Q}$ and thus $g_{S} \leqslant f$.

Finally, since $p<p^{\prime}$ it follows that

$$
\begin{aligned}
& g_{S}(p,-)=g_{0}(p,-) \vee\left(\underset{r^{\prime} \geq 0}{\vee} \underset{r^{\prime}<r^{\prime \prime}<p^{\prime}-p+r^{\prime}}{\bigvee} g_{0}\left(p-r^{\prime}, p^{\prime}-r^{\prime \prime}\right) \wedge h_{S}\left(\frac{r^{\prime}}{r^{\prime \prime}},-\right)\right) \\
& \quad \geq g_{0}(p,-) \vee\left(\bigvee_{r^{\prime} \geq 0} \underset{r^{\prime}<r^{\prime \prime}<p^{\prime}-p+r^{\prime}}{\vee} g_{0}\left(p-r^{\prime}, p^{\prime}-r^{\prime \prime}\right) \wedge h_{S}(-, 1)^{*}\right) \\
& \quad=g_{0}(p,-) \vee\left(\bigvee_{r^{\prime} \geq 0}^{\vee} g_{0}\left(p-r^{\prime}, p^{\prime}-r^{\prime}\right) \wedge S\right)=g_{0}(p,-) \vee\left(g_{0}\left(-, p^{\prime}\right) \wedge S\right) \\
& \quad=\left(g_{0}(p,-) \vee g_{0}\left(-, p^{\prime}\right)\right) \wedge\left(g_{0}(p,-) \vee S\right)=g_{0}(p,-) \vee S \geq S
\end{aligned}
$$

and thus $S \leqslant g_{S}(p,-) \leqslant \bigvee\{g(p,-) \mid g \in \mathrm{C}(L)$ and $g \leqslant f\}$. Hence

$$
\begin{aligned}
& \overline{f\left(p^{\prime},-\right)} \leqslant \bigvee\{g(p,-) \mid g \in \mathrm{C}(L) \text { and } g \leqslant f\} \quad \text { and } \\
& f^{\circ}(p,-)=\bigvee_{p^{\prime}>p} \overline{f\left(p^{\prime},-\right)} \leqslant \bigvee\{g(p,-) \mid g \in \mathrm{C}(L) \text { and } g \leqslant f\} .
\end{aligned}
$$

But from [20, Lemma 3.3] we know that

$$
\bigvee\{g(p,-) \mid g \in \mathrm{C}(L) \text { and } g \leqslant f\}=(\bigvee\{g \in \mathrm{C}(L) \mid g \leqslant f\})(p,-) .
$$

Hence $f^{\circ} \leqslant \bigvee\{g \in \mathrm{C}(L) \mid g \leqslant f\}$.

Corollary 4.2. Let $L$ be a completely regular frame and $f \in \mathrm{F}^{*}(L)$. Then:

(1) $f^{\circ}=\bigvee\left\{g \in \mathrm{C}^{*}(L) \mid g \leqslant f\right\}$.

(2) $f^{-}=\bigwedge\left\{g \in \mathrm{C}^{*}(L) \mid f \leqslant g\right\}$.

Proof. (1) Let $f \in \mathrm{F}^{*}(L)$ and $p, q \in \mathbb{Q}$ be such that $\boldsymbol{p} \leqslant f \leqslant \boldsymbol{q}$. Note that $g \vee \boldsymbol{p} \in \mathrm{C}^{*}(L)$ for any $g \in \mathrm{C}(L)$ such that $g \leq f$, since $\boldsymbol{p} \leq g \vee \boldsymbol{p} \leq \boldsymbol{q}$. Then, by Lemma 4.1 we have that

$$
\begin{aligned}
f^{\circ} & =\bigvee\{g \in \mathrm{C}(L) \mid g \leqslant f\} \leqslant \bigvee\{g \vee \boldsymbol{p} \mid g \in \mathrm{C}(L) \text { and } g \leqslant f\} \\
& \leqslant \bigvee\left\{g^{\prime} \in \mathrm{C}^{*}(L) \mid g^{\prime} \leqslant f\right\} .
\end{aligned}
$$

The converse inequality is trivial and (2) follows dually.

All this allows to extend the classical notions of lower and upper normal semicontinuous real functions on a topological space (due to Dilworth [11, Def. 3.2], see also [28]) into the pointfree setting: 
Definition 4.3. (Cf. [21]) An $f \in \mathrm{F}(L)$ is normal lower semicontinuous if

$$
f^{-} \in \mathrm{F}(L) \quad \text { and } \quad f^{-\circ}=f
$$

dually, $f$ is normal upper semicontinuous if

$$
f^{\circ} \in \mathrm{F}(L) \quad \text { and } \quad f^{\circ-}=f .
$$

We denote by $\operatorname{NLSC}(L)$ and $\operatorname{NUSC}(L)$ the classes of normal lower semicontinuous and normal upper semicontinuous members of $\mathrm{F}(L)$.

This is a slight refinement of our previous definition in [21], where we defined an $f \in \mathrm{F}(L)$ to be normal lower (resp. upper) semicontinuous just whenever $f^{-\circ}=f\left(\right.$ resp. $\left.f^{\circ-}=f\right)$, certainly inspired by the original definition of Dilworth in [11] — stating that a lower (resp. upper) semicontinuous real function $\varphi: X \rightarrow \mathbb{R}$ is normal if $\left(\varphi^{*}\right)_{*}=\varphi$ (resp. $\left.\left(\varphi_{*}\right)^{*}=\varphi\right)$. But it should be noted that Dilworth [11] was only dealing with bounded real functions. In the general case (of arbitrary, not necessarily bounded, real functions), it turns out that there are real functions satisfying $\left(\varphi^{*}\right)_{*}=\varphi$ such that $\varphi^{*}$ is not real (take, for instance, $\varphi: \mathbb{R} \rightarrow \mathbb{R}$ given by $\varphi(x)=0$ if $x \leqslant 0$ and $\varphi(x)=\frac{1}{x}$ if $x>0)$. So, when dealing with arbitrary real functions, the assumption that $\varphi^{*}$ and $\varphi_{*}$ be real (or, equivalently, $\varphi$ be locally bounded) is no longer redundant and needs to be added to the definition (as Mack and Johnson did in [28]).

Next result provides formulas for the double regularization of a locally bounded arbitrary real function. We direct the reader to [21, Lemma 3.4] for a proof of this result. Notice that in [21, Lemma 3.4] the notation $f \in \mathrm{F}^{b}(L)$ means that there exist $g \in \operatorname{LSC}(L)$ and $h \in \operatorname{USC}(L)$ such that $g \leqslant f \leqslant h$ and, by Corollary 3.6, this is equivalent to saying that $f$ is locally bounded.

Lemma 4.4. Let $f \in \mathrm{F}^{l b}(L)$. Then for every $p, q \in \mathbb{Q}$ we have:

(1) $f^{-\circ}(p,-)=\bigvee_{r>p} \overline{f(r,-)^{\circ}}$ and $f^{-\circ}(-, q)=\bigvee_{s<q}(\overline{f(-, s)})^{\circ}$.

(2) $f^{\circ-}(p,-)=\bigvee_{r>p}(\overline{f(r,-)})^{\circ}$ and $f^{\circ-}(-, q)=\bigvee_{s<q} \overline{f(-, s)^{\circ}}$.

Remark 4.5. Recall that a lower semicontinuous mapping $\varphi: X \rightarrow \mathbb{R}$ is normal if and only if it is locally bounded and

$$
\varphi^{-1}((p,+\infty))=\bigcup_{r>p} \operatorname{Int}\left(\overline{\varphi^{-1}((r,+\infty))}\right)
$$

for each $p \in \mathbb{Q}$. Given a lower semicontinuous mapping $\varphi: X \rightarrow \mathbb{R}$ and the corresponding lower semicontinuous real function $f_{\varphi}$ in $\mathrm{F}(\mathcal{O} X)$ introduced in Remark 2.4.1(3), $\varphi$ is normal lower semicontinuous if and only if $f_{\varphi}$ is normal lower semicontinuous. In fact, $\varphi \in \operatorname{LSC}^{l b}(X)$ if and only if $f_{\varphi} \in \operatorname{LSC}^{l b}(\mathcal{O} X)$ 
and moreover

$$
\begin{aligned}
\varphi=\left(\varphi^{*}\right)_{*} & \Longleftrightarrow \forall p \in \mathbb{Q} \quad \varphi^{-1}((p,+\infty))=\bigcup_{r>p} \operatorname{Int}\left(\overline{\varphi^{-1}((r,+\infty))}\right) \\
& \Longleftrightarrow \forall p \in \mathbb{Q} \quad \mathfrak{c}\left(\varphi^{-1}((p,+\infty))\right)=\bigvee_{r>p} \mathfrak{c}\left(\operatorname{Int}\left(\overline{\varphi^{-1}((r,+\infty))}\right)\right) \\
& \Longleftrightarrow \forall p \in \mathbb{Q} \quad \mathfrak{c}\left(\varphi^{-1}((p,+\infty))\right)=\bigvee_{r>p} \mathfrak{c}\left(\varphi^{-1}((r,+\infty))^{* *}\right) \\
& \Longleftrightarrow \forall p \in \mathbb{Q} \quad \mathfrak{c}\left(\varphi^{-1}((p,+\infty))\right)=\bigvee_{r>p} \overline{\mathfrak{c}\left(\varphi^{-1}((r,+\infty))\right)^{\circ}} \\
& \Longleftrightarrow \forall p \in \mathbb{Q} \quad f_{\varphi}(p,-)=\bigvee_{r>p} \overline{f_{\varphi}(r,-)^{\circ}}=\left(f_{\varphi}\right)^{-\circ}(p,-) \\
& \Longleftrightarrow f_{\varphi}=\left(f_{\varphi}\right)^{-\circ} .
\end{aligned}
$$

In conclusion, $\varphi$ is normal lower semicontinuous if and only if $f_{\varphi} \in \operatorname{NLSC}(\mathcal{O} X)$. Evidently, the dual situation for upper semicontinuous real functions also holds.

In the sequel, we shall be particularly interested in the following subclasses:

$$
\begin{aligned}
& \operatorname{NLSC}^{c b}(L)=\operatorname{NLSC}(L) \cap \mathrm{F}^{c b}(L), \quad \operatorname{NUSC}^{c b}(L)=\operatorname{NUSC}(L) \cap \mathrm{F}^{c b}(L), \\
& \operatorname{NLSC}^{*}(L)=\operatorname{NLSC}(L) \cap \mathrm{F}^{*}(L) \quad \text { and } \quad \operatorname{NUSC}^{*}(L)=\operatorname{NUSC}(L) \cap \mathrm{F}^{*}(L) .
\end{aligned}
$$

Remarks 4.6. (1) It follows from Proposition 3.3(3) and Corollary 3.6 that $\operatorname{NLSC}(L) \subseteq \operatorname{LSC}(L) \cap \mathrm{F}^{l b}(L)$ and $\operatorname{NUSC}(L) \subseteq \operatorname{USC}(L) \cap \mathrm{F}^{l b}(L)$.

(2) If $f \in \operatorname{NLSC}(L)$ then $f^{-} \in \operatorname{NUSC}(L)$; dually, if $f \in \operatorname{NUSC}(L)$ then $f^{\circ} \in \operatorname{NLSC}(L)$. Clearly, the operators $(\cdot)^{\circ}: \operatorname{NUSC}(L) \rightarrow \operatorname{NLSC}(L)$ and $(\cdot)^{-}: \operatorname{NLSC}(L) \rightarrow \operatorname{NUSC}(L)$ are inverse to each other and establish an orderisomorphism between the lattices $\operatorname{NLSC}(L)$ and $\operatorname{NUSC}(L)$. Note that there are also order-isomorphisms between the lattices $\operatorname{NLSC}^{c b}(L)$ and $\operatorname{NUSC}^{c b}(L)$, and $\operatorname{NLSC}^{*}(L)$ and $\operatorname{NUSC}(L)^{*}$.

(3) Given $f \in \operatorname{NLSC}(L)$ it is straightforward to check that $-f \in \operatorname{NUSC}(L)$ and hence that $-(\cdot)$ is a dual order-isomorphism between the lattices $\operatorname{NLSC}(L)$ and $\operatorname{NUSC}(L)$. When restricted to $\operatorname{NLSC}^{c b}(L)$ (resp. $\operatorname{NLSC}^{*}(L)$ ) it becomes a dual isomorphism from $\operatorname{NLSC}^{c b}(L)$ onto $\operatorname{NUSC}^{c b}(L)$ (resp. from $\operatorname{NLSC}^{*}(L)$ onto $\left.\operatorname{NUSC}^{*}(L)\right)$.

(4) The classical characteristic functions of subsets of a space have the following pointfree counterpart: for each complemented $S \in \mathcal{S}(L)$,

$$
\sigma(p)=1 \text { if } p<0, \quad \sigma(p)=S^{*} \text { if } 0 \leqslant p<1, \quad \sigma(p)=0 \text { if } p \geq 1
$$

is a scale describing a real function $\chi_{S} \in \mathrm{F}^{*}(L)$, called the characteristic function of $S$. Specifically, $\chi_{S}$ is defined for each $p \in \mathbb{Q}$ by

$$
\chi_{S}(p,-)=\left\{\begin{array}{ll}
1 & \text { if } p<0, \\
S^{*} & \text { if } 0 \leqslant p<1, \\
0 & \text { if } p \geq 1,
\end{array} \quad \text { and } \quad \chi_{S}(-, p)= \begin{cases}0 & \text { if } p \leqslant 0, \\
S & \text { if } 0<p \leqslant 1, \\
1 & \text { if } p>1\end{cases}\right.
$$

Then we have: 
(1) $\chi_{S} \in \operatorname{LSC}^{*}(L)$ iff $S$ is open and $\chi_{S} \in \operatorname{USC}^{*}(L)$ iff $S$ is closed.

(2) $\chi_{S} \in \mathrm{C}^{*}(L)$ iff $S$ is clopen.

(3) $\left(\chi_{S}\right)^{\circ}=\chi_{S^{\circ}}$ and $\left(\chi_{S}\right)^{-}=\chi_{\bar{S}}$.

(4) $\left(\chi_{\mathfrak{o}(a)}\right)^{\circ}=\chi_{\mathfrak{o}(a)},\left(\chi_{\mathfrak{c}(a)}\right)^{\circ}=\chi_{\mathfrak{o}\left(a^{*}\right)},\left(\chi_{\mathfrak{c}(a)}\right)^{-}=\chi_{\mathfrak{c}(a)}$ and $\left(\chi_{\mathfrak{o}(a)}\right)^{-}=\chi_{\mathfrak{c}\left(a^{*}\right)}$.

(5) $\chi_{\mathfrak{o}(a)} \in \operatorname{NLSC}^{*}(L)$ iff $a=a^{* *}$ iff $\chi_{\mathfrak{c}(a)} \in \operatorname{NUSC}^{*}(L)$.

We shall also need the following result:

Proposition 4.7. Let $\varnothing \neq \mathcal{F} \subseteq \operatorname{NLSC}(L)$. Then the join $\bigvee \mathcal{F}$ exists in $\overline{\mathrm{F}}(L)$.

Proof. Let $\sigma(p)=\bigvee_{f \in \mathcal{F}} f(p,-)$ for every $p \in \mathbb{Q}$. Since $\mathcal{F} \subseteq \operatorname{NLSC}(L)$, it follows from Lemma 4.4(1) that

$$
\sigma(p)=\bigvee_{f \in \mathcal{F}} f^{-\circ}(p,-)=\bigvee_{f \in \mathcal{F}} \bigvee_{r>p} \overline{f(r,-)^{\circ}}
$$

for each $p \in \mathbb{Q}$. The map $\sigma$ is clearly antitone. Since each $\sigma(p)$ is a closed sublocale (hence complemented), it follows from Remark 2.5 that $\sigma$ is an extended scale in $\mathcal{S}(L)$. Thus it determines a real function $g$ in $\overline{\mathrm{F}}(L)$ given by

$$
g(p,-)=\bigvee_{r>p} \sigma(r) \text { and } g(-, q)=\bigvee_{r<q} \sigma(r)^{*}, \quad p, q \in \mathbb{Q} .
$$

We claim that $g$ is the join of $\mathcal{F}$ in $\overline{\mathrm{F}}(L)$ :

- For each $f \in \mathcal{F}, f \leqslant g$, that is, $f(p,-) \leqslant g(p,-)$ for every $p \in \mathbb{Q}$ :

$$
\begin{aligned}
g(p,-) & =\bigvee_{r>p} \sigma(r)=\bigvee_{r>p} \bigvee_{f \in \mathcal{F}} f(r,-)=\bigvee_{f \in \mathcal{F}} \bigvee_{r>p} f(r,-) \\
& =\bigvee_{f \in \mathcal{F}} f(p,-) \geq f(p,-)
\end{aligned}
$$

- If $f \leqslant h$ for every $f \in \mathcal{F}$ and $h \in \overline{\mathrm{F}}(L)$, then $g \leqslant h$, that is, $g(p,-) \leqslant$ $h(p,-)$ for every $p \in \mathbb{Q}$ :

$$
g(p,-)=\bigvee_{f \in \mathcal{F}} f(p,-) \leqslant h(p,-) .
$$

Proposition 4.8. Let $f \in \mathrm{F}(L)$. The following hold:

(1) If $f \in \mathrm{F}^{c b}(L)$ then $f^{-\circ} \in \mathrm{NLSC}^{c b}(L)$.

(2) If $f \in \mathrm{F}^{*}(L)$ then $f^{-\circ} \in \operatorname{NLSC}^{*}(L)$.

Proof. (1) Choose $f \in \mathrm{F}^{c b}(L)$ and $h_{1}, h_{2} \in \mathrm{C}(L)$ such that $h_{1} \leqslant f \leqslant h_{2}$. By Proposition 3.3(4) and Corollary 3.4(1) it follows that

$$
h_{1}=h_{1}^{-} \leqslant f^{-} \leqslant h_{2}^{-}=h_{2} \quad \text { and } \quad h_{1}=h_{1}^{-\circ} \leqslant f^{-\circ} \leqslant h_{2}^{-\circ}=h_{2},
$$

which, together with Proposition 3.3(5), imply that $f^{-\circ} \in \operatorname{NLSC}^{c b}(L)$.

(2) This follows in a similar fashion as (1).

Now, we need to introduce a weak variant of the notion of a cb-frame:

Definition 4.9. A frame $L$ is a weak $c b$-frame if each locally bounded, lower semicontinuous real function on $L$ is bounded above by a continuous real function. 
We note that cb-frames and weakly cb-frames have also been considered by T. Dube [12, Definition 4.5] under different names (namely tower cozshrinkable and weakly tower coz-shrinkable) as the pointfree counterparts of the cb-spaces and weak cb-spaces of Mack and Johnson [28]. In [13], weakly tower coz-shrinkable frames are called weak-cb. Our definitions above are different, closer to the classical formulations but easily seen to be equivalent to Dube's ones.

Proposition 4.10. The following are equivalent for a frame $L$ :

(1) $L$ is weak $c b$.

(2) Every upper semicontinuous and locally bounded real function on $L$ is bounded below by a continuous real function.

(3) Every normal upper semicontinuous real function $f$ on $L$ is bounded above by a continuous real function.

(4) Every normal lower semicontinuous real function $f$ on $L$ is bounded below by a continuous real function.

(5) $\operatorname{LSC}^{c b}(L)=\operatorname{LSC}^{l b}(L)$.

(6) $\operatorname{USC}^{c b}(L)=\mathrm{USC}^{l b}(L)$.

(7) $\operatorname{NUSC}^{c b}(L)=\operatorname{NUSC}(L)$.

(8) $\operatorname{NLSC}^{c b}(L)=\operatorname{NLSC}(L)$.

Proof. (1) $\Longleftrightarrow(2)$ and $(3) \Longleftrightarrow(4)$ are clear since $f \in \operatorname{LSC}^{l b}(L)$ if and only if $-f \in \operatorname{USC}^{l b}(L)$ and $f \in \operatorname{NLSC}(L)$ if and only if $-f \in \operatorname{NUSC}(L)$.

$(1) \Longrightarrow(3)$ : Let $f \in \operatorname{NUSC}(L)$. If follows from Corollary 3.6 that $f^{\circ} \in$ $\operatorname{LSC}^{l b}(L)$. The hypothesis says there is a $g \in \mathrm{C}(L)$ such that $f^{\circ} \leqslant g$. Hence $f=f^{\circ-} \leqslant g^{-}=g$.

$(4) \Longrightarrow(1)$ : Let $f \in \operatorname{LSC}^{l b}(L)$. If follows from Corollary 3.6 that $f^{-}, f^{-\circ-} \in$ $\mathrm{F}(L)$. Moreover, $f^{-\circ-}=f^{-}$and so $f^{-} \in \operatorname{NUSC}(L)$. By the hypothesis there is a $g \in \mathrm{C}(L)$ such that $f^{-} \leqslant g$. Hence $f \leqslant f^{-} \leqslant g$.

$(5) \Longrightarrow(1),(6) \Longrightarrow(2),(7) \Longrightarrow(3)$ and $(8) \Longrightarrow(4)$ are obvious.

$(1) \Longrightarrow(5)$ : Let $f \in \operatorname{LSC}^{l b}(L)$. Then $-f^{-} \in \operatorname{LSC}^{l b}(L)$. By the hypothesis (applied to both $f$ and $-f^{-}$) there exist $g_{1}, g_{2} \in \mathrm{C}(L)$ such that $g_{1} \leqslant f$ and $g_{2} \leqslant-f^{-}$. Hence $g_{1} \leqslant f \leqslant f^{-} \leqslant-g_{2}$.

$(2) \Longrightarrow(6)$ is dual to $(1) \Longrightarrow(5)$.

$(3) \Longrightarrow(7)$ : Let $f \in \operatorname{NUSC}(L)$. Then, by Remark 4.6(2), $-f^{\circ} \in \operatorname{NUSC}(L)$. The hypothesis says there are $g_{1}, g_{2} \in \mathrm{C}(L)$ such that $f \leqslant g_{1}$ and $-f^{\circ} \leqslant g_{2}$. Hence $-g_{2} \leqslant f^{\circ} \leqslant f \leqslant g_{1}$.

$(4) \Longrightarrow(8)$ is dual to $(3) \Longrightarrow(7)$.

The careful reader will observe readily enough that in view of Proposition 4.10 and Remarks 2.4.1(3), 4.5, a topological space $X$ is a weak cb-space if and only if the frame $\mathcal{O} X$ is weak cb.

It also follows immediately from Proposition 4.10 (now using [21, Corollary 3.7$]$ ) that the class of weak cb-frames includes extremally disconnected frames. 


\section{The normal completion of $\mathrm{C}(L)$ and $\mathrm{C}^{*}(L)$}

We follow [32, Section 1.3] for the terminology on completions of a poset. Recall from there that a completion of $P$ is a pair $(C, \varphi)$ where $C$ is a complete lattice and $\varphi: P \rightarrow C$ is a join- and meet-dense embedding (that is, each element of $C$ is a join of elements from $\varphi[P]$, and dually each element of $C$ is a meet of elements from $\varphi[P])$.

Given a poset $P=(P, \leqslant)$, we denote by $\top$ and $\perp$ (in case they exist) the top and bottom elements of $P$, respectively. Given $A \subseteq P$, let $A^{u}$ (resp. $A^{l}$ ) denote the set of all upper (resp. lower) bounds of $A$ :

$A^{u}=\{x \in P \mid y \leqslant x$ for all $y \in A\}$ and $A^{l}=\{x \in P \mid x \leqslant y$ for all $y \in A\}$.

For any $A, B \subseteq P$, we have:

(1) $A^{u}$ is an upper set and $A^{l}$ is a lower set.

(2) $A \subseteq A^{u l} \cap A^{l u}$.

(3) If $A \subseteq B$ then $A^{u} \supseteq B^{u}$ and $A^{l} \supseteq B^{l}$.

(4) $A^{u l u}=A^{u}$ and $A^{l u l}=A^{l}$.

The MacNeille completion (or normal completion) of $P$ is the complete lattice

$$
M(P)=\left\{A \subseteq P \mid A^{u l}=A\right\}
$$

ordered by set inclusion, with $\varphi(a)=\{a\}^{l}$ for every $a \in P$. The top element of $M(P)$ is the whole poset $P$. On the other hand, the bottom element of $M(P)$ is the subset $\{\perp\}$ in case $P$ has a bottom element $\perp$, and $\varnothing$ otherwise.

Sometimes a weaker kind of completeness is more useful: a poset $(P, \leqslant)$ is Dedekind (order) complete (or conditionally complete) if every non-void subset $A$ of $P$ which is bounded from above has a supremum in $P$ (and then, in particular, every non-void subset $B$ of $P$ which is bounded from below will have a infimum in $P$ ). Of course, being complete is equivalent to Dedekind complete plus the existence of top and bottom elements. A Dedekind completion (or conditional completion) of $P$ is a join- and meet-dense embedding $\varphi: P \rightarrow D(P)$ in a Dedekind complete poset $D(P)$. The Dedekind completion is slightly smaller than the MacNeille completion: it can be obtained from $M(P)$, in case $P$ is directed, just by removing its top and bottom elements. In other words,

$$
D(P)=\left\{A \subseteq P \mid A^{u l}=A \text { and }\{\perp\} \neq A \neq P\right\}
$$

in case $P$ has a bottom element $\perp$ and

$$
D(P)=\left\{A \subseteq P \mid A^{u l}=A \text { and } \varnothing \neq A \neq P\right\}
$$

if $P$ has no bottom element.

Next we shall prove that the Dedekind completion $D(\mathrm{C}(L))$ of $\mathrm{C}(L)$ is isomorphic with $\mathrm{NLSC}^{c b}(L)$ (and consequently, by Remark 4.6(2), also with $\left.\operatorname{NUSC}^{c b}(L)\right)$. 
In order to describe $D(\mathrm{C}(L))$ there is no loss of generality if we restrict ourselves to completely regular frames (see the discussion in [6, Section 2]).

Theorem 5.1. Let $L$ be a completely regular frame. The map

$$
\Phi: D(\mathrm{C}(L)) \rightarrow \mathrm{NLSC}^{c b}(L) \quad \text { defined by } \Phi(\mathcal{A})=(\bigvee \mathcal{A})^{-\circ}
$$

(where $\bigvee \mathcal{A}$ denotes the supremum of $\mathcal{A}$ in $\overline{\mathrm{F}}(L)$ ) is a lattice isomorphism, with inverse

$$
\Psi: \operatorname{NLSC}^{c b}(L) \rightarrow D(\mathrm{C}(L)) \text { given by } \Psi(f)=\{g \in \mathrm{C}(L) \mid g \leqslant f\} .
$$

Proof. (1) $\Phi$ is well defined: Let $\mathcal{A} \in D(\mathrm{C}(L))$. We first note that since $\mathrm{C}(L)$ has no bottom element,

$$
D(\mathrm{C}(L))=\left\{\mathcal{A} \subseteq \mathrm{C}(L) \mid \mathcal{A}^{u l}=\mathcal{A} \text { and } \varnothing \neq \mathcal{A} \neq \mathrm{C}(L)\right\}
$$

and so $\mathcal{A} \neq \varnothing$. On the other hand, $\mathcal{A}^{u} \neq \varnothing$ (otherwise $\mathcal{A}=\mathcal{A}^{u l}=\mathrm{C}(L)$ ).

Let $f \in \mathcal{A}$ and $g \in \mathcal{A}^{u}$. The join $\bigvee \mathcal{A}$ exists in $\overline{\mathrm{F}}(L)$ by Proposition 4.7 and satisfies $f \leqslant \bigvee \mathcal{A} \leqslant g$, hence $\bigvee \mathcal{A} \in \mathrm{F}^{c b}(L)$. Then, by Proposition 4.8(1), $(\bigvee \mathcal{A})^{-\circ} \in \operatorname{NLSC}^{c b}(L)$.

(2) $\Psi$ is well defined: First note that since $f \in \mathrm{F}^{c b}(L)$, there exists a $g \in \mathrm{C}(L)$ such that $g \leqslant f$. Hence $\{g \in \mathrm{C}(L) \mid g \leqslant f\} \neq \varnothing$. Also, $\{g \in \mathrm{C}(L) \mid$ $g \leqslant f\} \neq \mathrm{C}(L)$ (since $\mathrm{C}(L)$ has no top element). Moreover, given $h \in \mathrm{C}(L)$, we have by Lemma $4.1(1)$

$$
\begin{aligned}
h \in\{g \in \mathrm{C}(L) \mid g \leqslant f\}^{u} & \Longleftrightarrow g \leqslant h \text { for all } g \in \mathrm{C}(L) \text { such that } g \leqslant f \\
& \Longleftrightarrow f=f^{\circ}=\bigvee\{g \in \mathrm{C}(L) \mid g \leqslant f\} \leqslant h .
\end{aligned}
$$

Then, by Lemma 4.1(2) we have, for each $h^{\prime} \in \mathrm{C}(L)$,

$$
\begin{aligned}
h^{\prime} \in\{g \in \mathrm{C}(L) \mid g \leqslant f\}^{u l} & \Longleftrightarrow h^{\prime} \leqslant h \text { for all } h \in\{g \in \mathrm{C}(L) \mid g \leqslant f\}^{u} \\
& \Longleftrightarrow h^{\prime} \leqslant h \text { for all } h \in \mathrm{C}(L) \text { such that } f \leqslant h \\
& \Longleftrightarrow h^{\prime} \leqslant \bigwedge\{h \in \mathrm{C}(L) \mid f \leqslant h\}=f^{-} \\
& \Longleftrightarrow h^{\prime}=h^{\prime} \leqslant f^{-\circ}=f .
\end{aligned}
$$

Hence $\{g \in \mathrm{C}(L) \mid g \leqslant f\}^{u l}=\{g \in \mathrm{C}(L) \mid g \leqslant f\}$.

(3) Both $\Phi$ and $\Psi$ are order-preserving: Choose $\mathcal{A}, \mathcal{B} \in D(\mathrm{C}(L))$ such that $\mathcal{A} \subseteq \mathcal{B}$. Then $\bigvee \mathcal{A} \leqslant \bigvee \mathcal{B}$ and so $(\bigvee \mathcal{A})^{-\circ} \leqslant(\bigvee \mathcal{B})^{-\circ}$, i.e., $\Phi(\mathcal{A}) \leqslant \Phi(\mathcal{B})$ Conversely, let $f, g \in \operatorname{NLSC}^{c b}(L)$ satisfying $f \leqslant g$. Then

$$
\Psi(f)=\{h \in \mathrm{C}(L) \mid h \leqslant f\} \subseteq\{h \in \mathrm{C}(L) \mid h \leqslant g\}=\Psi(g) .
$$

(4) $\Phi$ is a bijection with inverse $\Psi$ : Let $f \in \operatorname{NLSC}^{c b}(L)$. By Lemma 4.1(1),

$$
\begin{aligned}
\Phi(\Psi(f)) & =\Phi(\{g \in \mathrm{C}(L) \mid g \leqslant f\})=(\bigvee\{g \in \mathrm{C}(L) \mid g \leqslant f\})^{-\circ} \\
& =\left(f^{\circ}\right)^{-\circ}=f^{-\circ}=f .
\end{aligned}
$$


On the other hand, given $\mathcal{A} \in D(\mathrm{C}(L))$ and $g \in \mathrm{C}(L)$, we have (by Lemma 4.1(2) and since $g=g^{\circ}$ )

$$
\begin{aligned}
g \leqslant(\bigvee \mathcal{A})^{-\circ} & \Longleftrightarrow g \leqslant(\bigvee \mathcal{A})^{-}=\bigwedge\{h \in \mathrm{C}(L) \mid \bigvee \mathcal{A} \leqslant h\} \\
& \Longleftrightarrow g \leqslant \bigwedge\left\{h \in \mathrm{C}(L) \mid h \in \mathcal{A}^{u}\right\} \Longleftrightarrow g \in \mathcal{A}^{u l}=\mathcal{A} .
\end{aligned}
$$

Hence

$$
\Psi(\Phi(\mathcal{A}))=\Psi\left((\bigvee \mathcal{A})^{-\circ}\right)=\left\{g \in \mathrm{C}(L) \mid g \leqslant(\bigvee \mathcal{A})^{-\circ}\right\}=\mathcal{A}
$$

The preceding theorem (together with Proposition 4.10) leads immediately to the following:

Corollary 5.2. For any completely regular, weak cb-frame $L$, the Dedekind completion $D(\mathrm{C}(L))$ of $\mathrm{C}(L)$ is isomorphic with $\mathrm{NLSC}(L)$, as well as with $\operatorname{NUSC}(L)$.

Note that by Remark 4.5 this generalizes a classical result of Horn [22, Theorem 11].

It also follows from Theorem 5.1 that $\operatorname{NLSC}^{c b}(L)$ is Dedekind complete. For the sake of completeness, we present here a direct proof of this fact. First we will need the following lemma.

Lemma 5.3. If $f \in \operatorname{NLSC}^{c b}(L)$ then $-f^{-} \in \operatorname{NLSC}^{c b}(L)$.

Proof. Since there exist $h_{1}, h_{2} \in \mathrm{C}(L)$ such that $h_{1} \leqslant f \leqslant h_{2}$, it follows by Proposition 3.3(4) and Corollary 3.4(1) that

$$
-h_{2}=\left(-h_{2}\right)^{-} \leqslant-f^{-} \leqslant\left(-h_{1}\right)^{-}=-h_{1},
$$

and so $-f^{-} \in \mathrm{F}^{c b}(L)$. On the other hand, $\left(-f^{-}\right)^{-}=-f^{\circ-}=-f \in \mathrm{F}(L)$. Since $f^{-}=f^{-\circ-}$, we also have

$$
\left(-f^{-}\right)^{-\circ}=\left(-f^{-\circ-}\right)^{-\circ}=-f^{-\circ-\circ-}=-f^{-} .
$$

Hence $-f^{-} \in \operatorname{NLSC}^{c b}(L)$.

Proposition 5.4. $\operatorname{NLSC}^{c b}(L)$ is Dedekind complete.

Proof. Let $\varnothing \neq \mathcal{F} \subseteq \operatorname{NLSC}^{c b}(L)$ and $f^{\prime} \in \operatorname{NLSC}^{c b}(L)$ such that

$$
f \leqslant f^{\prime} \text { for all } f \in \mathcal{F} .
$$

By Proposition 4.7 we know that the join $g=\bigvee \mathcal{F}$ exists in $\overline{\mathrm{F}}(L)$. Then $f \leqslant$ $g \leqslant f^{\prime}$ for each $f \in \mathcal{F}$ and so there exist $h_{1}, h_{2} \in \mathrm{C}(L)$ such that $h_{1} \leqslant g \leqslant h_{2}$, i.e., $g \in \mathrm{F}^{c b}(L)$. By Proposition 4.8(1) ,it follows that $g^{-\circ} \in \operatorname{NLSC}^{c b}(L)$. We claim that $g^{-\circ}$ is the join of $\mathcal{F}$ in $\operatorname{NLSC}^{c b}(L)$ :

- $f \leqslant g$ for every $f \in \mathcal{F}$ and so it follows by Proposition 3.3(4) and Corollary 3.4(1), that $f=f^{-\circ} \leqslant g^{-\circ}$ for every $f \in \mathcal{F}$.

- If $g^{\prime} \in \operatorname{NLSC}^{c b}(L)$ is such that $f \leqslant g^{\prime}$ for every $f \in \mathcal{F}$, then $g \leqslant g^{\prime}$ and thus (again by Proposition 3.3(4)) $g^{-\circ} \leqslant\left(g^{\prime}\right)^{-\circ}=g^{\prime}$. 
Now let $\varnothing \neq \mathcal{F} \subseteq \operatorname{NLSC}^{c b}(L)$ and $f^{\prime} \in \operatorname{NLSC}^{c b}(L)$ such that

$$
f^{\prime} \leqslant f \quad \text { for all } f \in \mathcal{F} .
$$

It follows from Lemma 5.3 that $\varnothing \neq \mathcal{G}=\left\{-f^{-} \mid f \in \mathcal{F}\right\} \subseteq \operatorname{NUSC}^{c b}(L)$, $-f^{\prime-} \in \operatorname{NLSC}^{c b}(L)$ and

$$
-f^{-} \leqslant-f^{\prime-} \text { for all } f \in \mathcal{F} .
$$

By the result above we have that $(\bigvee \mathcal{G})^{-\circ}$ is the join of $\mathcal{G}$ in $\operatorname{NLSC}^{c b}(L)$. We claim that $-(\bigvee \mathcal{G})^{-0-}$ is the meet of $\mathcal{F}$ in $\operatorname{NLSC}^{c b}(L)$ :

- $-(\bigvee \mathcal{G})^{-0-} \in \operatorname{NLSC}^{c b}(L)$ by Lemma 5.3.

- Since $-f^{-} \leqslant(\bigvee \mathcal{G})^{-\circ}$ for each $f \in \mathcal{F}$ we have

$$
-f=-f^{-\circ}=\left(-f^{-}\right)^{-} \leqslant(\bigvee \mathcal{G})^{-\circ-}
$$

and therefore $-(\bigvee \mathcal{G})^{-0-} \leqslant f$ for every $f \in \mathcal{F}$.

- Let $g^{\prime} \in \operatorname{NLSC}^{c b}(L)$ satisfying $g^{\prime} \leqslant f$ for each $f \in \mathcal{F}$. It follows that $-f^{-} \leqslant-g^{-}$for each $f \in \mathcal{F}$ with $-g^{\prime-} \in \operatorname{NLSC}^{c b}(L)$ and consequently $(\bigvee \mathcal{G})^{-\circ} \leqslant-g^{\prime-}$. To finish off the proof observe that

$$
g^{\prime}=g^{\prime-\circ} \leqslant\left(-(\bigvee \mathcal{G})^{-\circ}\right)^{\circ}=-(\bigvee \mathcal{G})^{-\circ-} .
$$

The bounded case. It is a straightforward exercise to adapt the proof of Theorem 5.1 to the case of bounded real functions. We then conclude the following:

Theorem 5.5. Let $L$ be a completely regular frame. The Dedekind completion $D\left(\mathrm{C}^{*}(L)\right)$ of $\mathrm{C}^{*}(L)$ is isomorphic with $\operatorname{NLSC}^{*}(L)$.

This generalizes Dilworth [11, Theorem 4.1] for spaces.

The case of extremally disconnected frames. Recall that a frame $L$ is said to be extremally disconnected if $a^{*} \vee a^{* *}=1$ for every $a \in L$ (equivalently, $L$ is extremally disconnected iff $a^{* *}$ is complemented for every $a \in L$ iff the closure of every open sublocale of $L$ is open iff the interior of every closed sublocale of $L$ is closed).

We first note the following:

Proposition 5.6. The following statements are equivalent for any frame $L$ :

(1) $L$ is extremally disconnected.

(2) $\operatorname{NLSC}(L)=\mathrm{C}(L)$.

(3) $\operatorname{NUSC}(L)=\mathrm{C}(L)$.

(4) $\operatorname{NLSC}^{*}(L)=\mathrm{C}^{*}(L)$.

(5) $\operatorname{NUSC}^{*}(L)=\mathrm{C}^{*}(L)$.

(6) $\operatorname{NLSC}^{c b}(L)=\mathrm{C}(L)$.

(7) $\operatorname{NUSC}^{c b}(L)=\mathrm{C}(L)$. 
Proof. (1) $\Longrightarrow(2)$ : Let $f \in \operatorname{NLSC}(L)$. Then, by Lemma 4.4 , for every $q \in \mathbb{Q}$ we have that

$$
f(-, q)=f^{-\circ}(-, q)=\bigvee_{s<q}(\overline{f(-, s)})^{\circ} .
$$

Since $L$ is extremally disconnected, it follows that $(\overline{f(-, s)})^{\circ}$ is a closed sublocale for any $s \in \mathbb{Q}$ and so $f(-, q)$ is closed for each $q \in \mathbb{Q}$, i.e., $f \in \operatorname{USC}(L)$. Hence $f \in \mathrm{C}(L)$.

$(2) \Longrightarrow(1)$ : For each $a \in L, \chi_{\mathfrak{o}\left(a^{* *}\right)} \in \operatorname{NLSC}(L)=\mathrm{C}(L)$ and so $\mathfrak{o}\left(a^{* *}\right)$ is a clopen sublocale, i.e., $a^{* *}$ is complemented.

The equivalences $(1) \Longleftrightarrow(3),(1) \Longleftrightarrow(4)$ and $(1) \Longleftrightarrow(5)$ follow similarly. Finally, the implications $(2) \Longrightarrow(6)$ and $(3) \Longrightarrow(7)$ are trivial while $(6) \Longrightarrow(1)$ follows from the fact that $\chi_{\mathfrak{o}\left(a^{* *}\right)}$ is indeed in $\operatorname{NLSC}(L)^{c b}=\mathrm{C}(L)$. Similarly for $(7) \Longrightarrow(1)$.

As an immediate corollary we get the following result from BanaschewskiHong [6]:

Corollary 5.7. ([6, Proposition 1]) The following are equivalent for any completely regular frame $L$ :

(1) $L$ is extremally disconnected.

(2) $\mathrm{C}(L)$ is Dedekind complete.

(3) $\mathrm{C}^{*}(L)$ is Dedekind complete.

\section{The completion as a function ring: bounded case}

In this section we will show that the Dedekind completion of the lattice of bounded continuous real functions on any completely regular frame is isomorphic to the lattice of all bounded continuous real functions on another suitably determined frame. The latter is a Boolean frame, namely the Booleanization $\mathfrak{B}(L)$ of $L$ [7], that is, the complete Boolean algebra of all regular elements $a=a^{* *}$.

Notation. Along the next two sections, for each real function $f$ and each $p \in \mathbb{Q}$ we shall denote the infima of the sublocales $f(p,-)$ and $f(-, p)$ by $f_{p}$ and $f^{p}$, respectively. In other words, $\mathfrak{c}\left(f_{p}\right)=\overline{f(p,-)}$ and $\mathfrak{c}\left(f^{p}\right)=\overline{f(-, p)}$.

Remarks 6.1. (1) Note that $0=f(p,-) \wedge f(-, p) \geq \overline{f(p,-)} \wedge \overline{f(-, p)}=$ $\mathfrak{c}\left(f_{p}\right) \wedge \mathfrak{c}\left(f^{p}\right)=\mathfrak{c}\left(f_{p} \wedge f^{p}\right)$ and thus $f_{p} \wedge f^{p}=0$ for every $p \in \mathbb{Q}$.

(2) If $f$ is locally bounded, then

$$
1=\bigvee_{p \in \mathbb{Q}} \overline{f(p,-)}=\bigvee_{p \in \mathbb{Q}} \mathfrak{c}\left(f_{p}\right)=\mathfrak{c}\left(\bigvee_{p \in \mathbb{Q}} f_{p}\right)
$$

and so $\bigvee_{p \in \mathbb{Q}} f_{p}=1$; similarly $\bigvee_{p \in \mathbb{Q}} f^{p}=1$

(3) If $f$ is lower semicontinuous (resp. upper semicontinuous) then, for each $p \in \mathbb{Q}$, we have $\bigvee_{r>p} f_{r}=f_{p}\left(\operatorname{resp} . \bigvee_{r<p} f^{r}=f^{p}\right)$ 
(4) If $f$ is normal lower semicontinuous then, by Lemma 4.4(1), $\mathfrak{c}\left(f_{p}\right)=$ $\bigvee_{r>p} \mathfrak{c}\left(\left(f_{r}\right)^{* *}\right)$ and therefore $\bigvee_{r>p}\left(f_{r}\right)^{* *}=f_{p}$. Dually, if $f$ is normal upper semicontinuous then $\bigvee_{s<q}\left(f^{s}\right)^{* *}=f^{q}$.

(5) Note also that if $f$ is continuous, the frame homomorphism $\varphi: \mathfrak{L}(\mathbb{R}) \rightarrow L$ such that $f=\mathfrak{c} \cdot \varphi$ is given precisely by $\varphi(p,-)=f_{p}$ and $\varphi(-, p)=f^{p}$ for each $p \in \mathbb{Q}$ (see Remark 2.4.1(2)).

Theorem 6.2. Let $L$ be a completely regular frame. The Dedekind completion of $\mathrm{C}^{*}(L)$ is isomorphic with $\mathrm{C}^{*}(\mathfrak{B}(L))$.

Proof. For each $f \in \operatorname{NLSC}^{*}(L)$ define $\sigma: \mathbb{Q} \rightarrow \mathfrak{B}(L)$ by $\sigma(r)=\left(f_{r}\right)^{* *}$ for every $r \in \mathbb{Q}$. The map $\sigma$ is trivially antitone and hence an extended scale in $\mathfrak{B}(L)$ by Remark 2.5. Moreover, since $f$ is bounded, there exist $p, q \in \mathbb{Q}$ such that $f(p, q)=1$. Then $f_{p}=1=f^{q}$,

$$
\bigvee_{r \in \mathbb{Q}} \sigma(r) \geq f_{p}=1 \quad \text { and } \bigvee_{r \in \mathbb{Q}} \sigma(r)^{*} \geq\left(f_{q}\right)^{*} \geq f^{q}=1
$$

Hence $\sigma$ is a scale in $\mathfrak{B}(L)$ and it then follows from (2.5.1) that the formulas

$$
\begin{aligned}
& \Phi(f)(p,-)=\bigvee_{r>p}^{\mathfrak{B}(L)}\left(f_{r}\right)^{* *}=\left(\bigvee_{r>p}^{L}\left(f_{r}\right)^{* *}\right)^{* *}=\left(\bigvee_{r>p}^{L} f_{r}\right)^{* *}=\left(f_{p}\right)^{* *} \text { and } \\
& \Phi(f)(-, q)=\bigvee_{s<q}^{\mathfrak{B}(L)}\left(f_{s}\right)^{*}=\left(\bigvee_{s<q}^{L}\left(f_{s}\right)^{*}\right)^{* *}
\end{aligned}
$$

determine a bounded continuous real function $\Phi(f)$ in $\mathfrak{B}(L)$. It is straightforward to check that the map $\Phi: \operatorname{NLSC}^{*}(L) \rightarrow \mathrm{C}^{*}(\mathfrak{B}(L))$ is order-preserving.

On the other hand, for each $g \in \mathrm{C}^{*}(\mathfrak{B}(L))$, let $\sigma: \mathbb{Q} \rightarrow \mathcal{S}(L)$ be given by $\sigma(r)=\mathfrak{c}(g(r,-))$ for every $r \in \mathbb{Q}$. The map $\sigma$ is trivially antitone and hence, by Remark 2.5, an extended scale in $\mathcal{S}(L)$. Moreover, since $g$ is bounded there exist $p, q \in \mathbb{Q}$ such that $g(p, q)=1$. Hence

$$
\bigvee_{r \in \mathbb{Q}} \sigma(r) \geq \mathfrak{c}(g(p,-))=\mathfrak{c}(1)=1 \quad \text { and } \quad \bigvee_{r \in \mathbb{Q}} \sigma(r)^{*} \geq \mathfrak{o}(g(q,-))=\mathfrak{o}(0)=1
$$

This shows that $\sigma$ is a scale in $\mathcal{S}(L)$ and it follows from (2.5.1) that the formulas

$$
\Psi(g)(p,-)=\bigvee_{r>p} \mathfrak{c}(g(r,-)) \text { and } \Psi(g)(-, q)=\bigvee_{s<q} \mathfrak{o}(g(s,-))
$$

determine a bounded lower semicontinuous real function $\Psi(g)$ in $L$. Moreover,

$$
\begin{aligned}
(\Psi(g))^{-\circ}(p,-) & =\bigvee_{r>p} \overline{\Psi(g)(r,-)^{\circ}}=\bigvee_{r>p} \mathfrak{c}\left(\bigvee_{s>r}^{L} g(s,-)\right)^{\circ} \\
& =\bigvee_{r>p} \mathfrak{c}\left(\left(\bigvee_{s>r}^{L} g(s,-)\right)^{* *}\right)=\bigvee_{r>p} \mathfrak{c}\left(\bigvee_{s>r}^{\mathfrak{B}(L)} g(s,-)\right) \\
& =\bigvee_{r>p} \mathfrak{c}(g(r,-))=\Psi(g)(p,-)
\end{aligned}
$$

for each $p \in \mathbb{Q}$. Hence $\Psi(g) \in \operatorname{NLSC}^{*}(L)$. Here again it is easily seen that the map $\Psi: \mathrm{C}^{*}(\mathfrak{B}(L)) \rightarrow \mathrm{NLSC}^{*}(L)$ is order-preserving. 
Finally, for each $f \in \operatorname{NLSC}^{*}(L), g \in \mathrm{C}^{*}(\mathfrak{B}(L))$ and $p \in \mathbb{Q}$, it follows from Remark 6.1(4) that

$$
\begin{aligned}
& \Psi(\Phi(f))(p,-)=\mathfrak{c}\left(\bigvee_{r>p} \Phi(f)(r,-)\right)=\mathfrak{c}\left(\bigvee_{r>p}\left(f_{r}\right)^{* *}\right)=\mathfrak{c}\left(f_{p}\right)=f(p,-) \quad \text { and } \\
& \Phi(\Psi(g))(p,-)=\left(\Psi(g)_{p}\right)^{* *}=\left(\bigvee_{r>p}^{L} g(r,-)\right)^{* *}=\bigvee_{r>p}^{\mathfrak{B}(L)} g(r,-)=g(p,-)
\end{aligned}
$$

and so $\Psi \cdot \Phi=1_{\mathrm{NLSC}^{*}(L)}$ and $\Phi \cdot \Psi=1_{\mathrm{C}^{*}(\mathfrak{B}(L))}$.

\section{The completion as a function ring: general case}

The preceding theorem has no counterpart for a general $\mathrm{C}(L)$ since there are frames $L$ (even spatial frames) for which the Dedekind completion of $\mathrm{C}(L)$ cannot be isomorphic to some $\mathrm{C}(M)$. In order to deal with the general case we shall need first to review briefly some basic notions and facts about frame homomorphisms and their right adjoints.

Given a frame homomorphism $h: L \rightarrow M$, let $h_{*}: M \rightarrow L$ denote its right adjoint, characterized by the condition $h(a) \leqslant b$ if and only if $a \leqslant h_{*}(b)$ for all $a \in L$ and $b \in M$. Obviously, $h$ is injective iff $h_{*} h=\mathrm{id}_{L}$ iff $h_{*}$ is surjective. In particular, if $h$ is injective then $h_{*}(0)=0$. We shall denote by $h_{*}[-]$ the image map $\mathcal{S}(M) \rightarrow \mathcal{S}(L)$ induced by $h_{*}$ (which sends each sublocale $S$ of $M$ to $\left.h_{*}[S]\right)$. This is a localic map [31,2.2].

Recall that $h$ is said to be

(1) closed if $h_{*}[-]$ preserves closed sublocales, that is, if $h_{*}[\mathfrak{c}(a)]=\mathfrak{c}\left(h_{*}(a)\right)$ for every $a \in M$,

(2) proper (also, perfect) if it is closed and $h_{*}$ preserves directed joins,

(3) an essential embedding if it is injective and $h_{*}(a)=0$ implies $a=0$ for each $a \in M$ (cf. [5, Lemma 1]).

Remark 7.1. In case $h_{*}$ preserves directed joins, then $h_{*}\left(a^{*}\right) \leqslant h_{*}(a)^{*}$. Indeed, $h_{*}\left(a^{*}\right)=h_{*}(\bigvee\{x \mid x \wedge a=0\})$ and the set $\{x \mid x \wedge a=0\}$ is clearly directed; hence

$$
h_{*}\left(a^{*}\right)=\bigvee\left\{h_{*}(x) \mid x \wedge a=0\right\} \leqslant \bigvee\left\{y \mid y \wedge h_{*}(a)=0\right\}=h_{*}(a)^{*} .
$$

Lemma 7.2. Let $h$ be an essential embedding. Then:

(1) For each $a \in M, h_{*}\left(a^{*}\right)=h_{*}(a)^{*}$. Consequently, $h_{*}\left(a^{* *}\right)=h_{*}(a)^{* *}$.

(2) For each $a \in M, h\left(h_{*}(a)\right)^{*}=a^{*}$. Consequently, $h\left(h_{*}(a)\right)^{* *}=a^{* *}$.

Proof. (1) First note that $h_{*}\left(a^{*}\right) \wedge h_{*}(a)=h_{*}(0)=0$ and thus $h_{*}\left(a^{*}\right) \leqslant h_{*}(a)^{*}$. On the other hand, fix an $a \in M$. Since $h_{*}$ is surjective there exists $x_{a} \in M$ such that $h_{*}(a)^{*}=h_{*}\left(x_{a}\right)$ and so $h_{*}\left(x_{a} \wedge a\right)=h_{*}\left(x_{a}\right) \wedge h_{*}(a)=0$. It then follows that $x_{a} \wedge a=0$ since $h$ is an essential embedding. Hence $x_{a} \leqslant a^{*}$ and $h_{*}(a)^{*}=h_{*}\left(x_{a}\right) \leqslant h_{*}\left(a^{*}\right)$. 
(2) The first inequality is immediate since $h\left(h_{*}(a)\right) \leqslant a$ and therefore $h\left(h_{*}(a)\right)^{*} \geq a^{*}$ for every $a \in M$. On the other hand, from (1) we have that

$$
\begin{aligned}
0 & =h_{*}(a) \wedge h_{*}(a)^{*}=h_{*}(a) \wedge h_{*}\left(h\left(h_{*}(a)\right)\right)^{*}=h_{*}(a) \wedge h_{*}\left(h\left(h_{*}(a)\right)^{*}\right) \\
& =h_{*}\left(a \wedge h\left(h_{*}(a)\right)^{*}\right)
\end{aligned}
$$

and therefore $a \wedge h\left(h_{*}(a)\right)^{*}=0$. Hence $h\left(h_{*}(a)\right)^{*} \leqslant a^{*}$ and finally observe that $a^{* *} \leqslant h\left(h_{*}(a)\right)^{* *}$.

We shall also make use of the following result, which is the version for completely regular frames, due to Chen [8], of a original result of Banaschewski [2] for compact regular frames (cf. [25, 26]):

Theorem 7.3. For every completely regular frame $L$, there exist a completely regular and extremally disconnected frame $\mathfrak{G}(L)$ and a proper essential embedding $\gamma_{L}: L \rightarrow \mathfrak{G}(L)$. Moreover, $\gamma_{L}$ is unique up to isomorphism.

The embedding $\gamma_{L}: L \rightarrow \mathfrak{G}(L)$ is usually called the Gleason cover (also Gleason envelope) of $L$.

Let $h: L \rightarrow M$ be a closed frame homomorphism and $f \in \operatorname{LSC}(M)$. For each $t \in \mathbb{Q}, f(t,-)=\mathfrak{c}\left(f_{t}\right)$ and so $h$ being closed implies that $h_{*}[f(t,-)]=$ $\mathfrak{c}\left(h_{*}\left(f_{t}\right)\right)$ for every $t \in \mathbb{Q}$. First, let us check that the composition $h_{*}[-]$. $f: \mathfrak{L}(\mathbb{R}) \rightarrow \mathcal{S}(L)$ establishes a real function whenever $h$ is a proper essential embedding.

Lemma 7.4. Let $h: L \rightarrow M$ be a closed frame homomorphism and $f \in$ $\operatorname{LSC}(M)$. The map $\sigma: \mathbb{Q} \rightarrow \mathcal{S}(L)$ given by

$$
\left.\sigma(p)=h_{*}[f(p,-)]\right)=\mathfrak{c}\left(h_{*}\left(f_{p}\right)\right)
$$

is an extended scale in $\mathcal{S}(L)$.

Proof. Let $p<q$. Then

$$
\sigma(p) \vee \sigma(q)^{*}=\mathfrak{c}\left(h_{*}\left(f_{p}\right)\right) \vee \mathfrak{o}\left(h_{*}\left(f_{q}\right)\right) \geq \mathfrak{c}\left(h_{*}\left(f_{p}\right)\right) \vee \mathfrak{o}\left(h_{*}\left(f_{p}\right)\right)=1
$$

It then follows from (2.5.1) that the formulas

$$
\begin{aligned}
& \left.h^{\leftarrow}(f)(p,-)=\bigvee_{r>p} h_{*}[f(r,-)]\right)=\bigvee_{r>p} \mathfrak{c}\left(h_{*}\left(f_{r}\right)\right) \quad \text { and } \\
& h^{\leftarrow}(f)(-, q)=\bigvee_{s<q}\left(h_{*}[f(s,-)]\right)^{*}=\bigvee_{s<q} \mathfrak{o}\left(h_{*}\left(f_{s}\right)\right)
\end{aligned}
$$

determine a real function $h^{\leftarrow}(f)$ in $\overline{\operatorname{LSC}}(L)$.

Clearly, $h^{\leftarrow}(\cdot)$ is monotone, that is, $f_{1} \leqslant f_{2}$ implies $h^{\leftarrow}\left(f_{1}\right) \leqslant h^{\leftarrow}\left(f_{2}\right)$.

Proposition 7.5. If $h: L \rightarrow M$ is a proper essential embedding and $f \in$ $\mathrm{C}(M)$, then $h^{\leftarrow}(f) \in \mathrm{NLSC}(L)$.

Proof. Since $h_{*}$ preserves directed joins, we have

$$
h^{\leftarrow}(f)(p,-)=\mathfrak{c}\left(h_{*}\left(\bigvee_{r>p} f_{r}\right)\right)=\mathfrak{c}\left(h_{*}\left(f_{p}\right)\right)=h_{*}\left[\mathfrak{c}\left(f_{p}\right)\right]
$$


for each $p \in \mathbb{Q}$.

We first prove that $h^{\leftarrow}(f)$ turns the defining relation (r5) into an identity in $\mathcal{S}(L)$. Indeed, since $h_{*}$ preserves directed joins, we have

$$
\bigvee_{p \in \mathbb{Q}} h^{\leftarrow}(f)(p,-)=\bigvee_{p \in \mathbb{Q}} \mathfrak{c}\left(h_{*}\left(f_{p}\right)\right)=\mathfrak{c}\left(h_{*}\left(\bigvee_{p \in \mathbb{Q}} f_{p}\right)\right)=\mathfrak{c}\left(h_{*}(1)\right)=1
$$

On the other hand, in order to prove that $h^{\leftarrow}(f)$ turns the defining relation (r6) into an identity in $\mathcal{S}(L)$, we proceed as follows. Since $h_{*}$ preserves meets we have that

$$
\mathfrak{c}\left(h_{*}\left(f_{t}\right)\right) \wedge \mathfrak{c}\left(h_{*}\left(f^{t}\right)\right)=\mathfrak{c}\left(h_{*}\left(f_{t} \wedge f^{t}\right)\right)=\mathfrak{c}\left(h_{*}(0)\right)=\mathfrak{c}(0)=0
$$

and consequently $\mathfrak{c}\left(h_{*}\left(f_{t}\right)\right) \wedge \mathfrak{c}\left(h_{*}\left(f^{t}\right)\right)=0$. Hence $\mathfrak{c}\left(h_{*}\left(f^{t}\right)\right) \leqslant \mathfrak{o}\left(h_{*}\left(f_{t}\right)\right)$. Finally observe that, since $h_{*}$ preserves directed joins and $f$ is locally bounded,

$$
\begin{aligned}
\bigvee_{q \in \mathbb{Q}} \overline{h^{\leftarrow}(f)(-, q)} & =\bigvee_{q \in \mathbb{Q}} \overline{\bigvee_{s<q} \mathfrak{o}\left(h_{*}\left(f_{s}\right)\right)} \geq \underset{q \in \mathbb{Q}}{\bigvee} \overline{\bigvee_{s<q} \mathfrak{c}\left(h_{*}\left(f^{s}\right)\right)}=\bigvee_{r \in \mathbb{Q}} \mathfrak{c}\left(h_{*}\left(f^{s}\right)\right) \\
& =\mathfrak{c}\left(h_{*}\left(\bigvee_{r \in \mathbb{Q}} f^{s}\right)\right)=\mathfrak{c}\left(h_{*}(1)\right)=\mathfrak{c}(1)=1 .
\end{aligned}
$$

Therefore $\bigvee_{q \in \mathbb{Q}} h^{\leftarrow}(f)(-, q) \geq \bigvee_{q \in \mathbb{Q}} \overline{h^{\leftarrow}(f)(-, q)}=1$ and $h^{\leftarrow}(f) \in \operatorname{LSC}(L)$.

Moreover, we have also proved that $h^{\leftarrow}(f)$ is locally bounded. Consequently, in order to demonstrate that $h^{\leftarrow}(f)$ is normal we only need to prove that $\left(h^{\leftarrow}(f)\right)^{-\circ}=h^{\leftarrow}(f)$. By Lemma 4.4, using Lemma 7.2(1) and Remark 6.1(4), we get, for each $p \in \mathbb{Q}$

$$
\begin{aligned}
\left(h^{\leftarrow}(f)\right)^{-\circ}(p,-) & =\bigvee_{r>p} \overline{h^{\leftarrow}(f)(r,-)^{\circ}}=\bigvee_{r>p} \overline{\mathfrak{c}\left(h_{*}\left(f_{r}\right)\right)^{\circ}}=\bigvee_{r>p} \mathfrak{c}\left(h_{*}\left(f_{r}\right)^{* *}\right) \\
& \left.=\bigvee_{r>p} \mathfrak{c}\left(h_{*}\left(\left(f_{r}\right)^{* *}\right)\right)\right)=\mathfrak{c}\left(h_{*}\left(\bigvee_{r>p}\left(f_{r}\right)^{* *}\right)\right) \\
& \left.\left.=\mathfrak{c}\left(h_{*}\left(f_{p}\right)\right)\right)=h^{\leftarrow}(f)(p,-)\right) .
\end{aligned}
$$

Proposition 7.6. Let $h: L \rightarrow M$ be a frame homomorphism with $M$ extremally disconnected. For each $g \in \operatorname{NLSC}(L)$ and $p, q \in \mathbb{Q}$ define

$$
h^{\rightarrow}(g)(p,-)=\bigvee_{r>p} \mathfrak{c}\left(h\left(g_{r}\right)^{* *}\right) \quad \text { and } \quad h^{\rightarrow}(g)(-, q)=\bigvee_{s<q} \mathfrak{c}\left(h\left(g_{s}\right)^{*}\right) .
$$

Then $h^{\rightarrow}(g) \in \mathrm{C}(M)$. Moreover, if $g_{1}, g_{2} \in \mathrm{NLSC}(L)$ are such that $g_{1} \leqslant g_{2}$ then $h \rightarrow\left(g_{1}\right) \leqslant h \rightarrow\left(g_{2}\right)$.

Proof. For each $g \in \operatorname{NLSC}(L)$ define $\sigma: \mathbb{Q} \rightarrow M$ by $\sigma(r)=h\left(g_{r}\right)^{* *}$ for every $r \in \mathbb{Q}$. Let $p<t<q$ in $\mathbb{Q}$. Since $M$ is extremally disconnected, we have

$$
\sigma(p) \vee \sigma(q)^{*}=h\left(g_{p}\right)^{* *} \vee h\left(g_{q}\right)^{*} \geq h\left(g_{t}\right)^{* *} \vee h\left(g_{t}\right)^{*}=1
$$

Since $g$ is locally bounded, it follows from Remark 6.1(2) that

$$
\bigvee_{p \in \mathbb{Q}} \sigma(p)=\bigvee_{p \in \mathbb{Q}} h\left(g_{p}\right)^{* *} \geq \bigvee_{p \in \mathbb{Q}} h\left(g_{p}\right)=h\left(\bigvee_{p \in \mathbb{Q}} g_{p}\right)=h(1)=1
$$


On the other hand, since $g_{p} \wedge g^{p}=0$, then $h\left(g_{p}\right) \wedge h\left(g^{p}\right)=0$ and thus $h\left(g^{p}\right) \leqslant$ $h\left(g_{p}\right)^{*}$ for every $p \in \mathbb{Q}$. Consequently, by Remark $6.1(2)$, we also get

$$
\bigvee_{p \in \mathbb{Q}} \sigma(p)^{*}=\bigvee_{p \in \mathbb{Q}} h\left(g_{p}\right)^{*} \geq \bigvee_{p \in \mathbb{Q}} h\left(g^{p}\right)=h\left(\bigvee_{p \in \mathbb{Q}} g^{p}\right)=h(1)=1
$$

Hence $\sigma$ is a scale in $M$.

It then follows from (2.5.1) and Remark 2.4.1(2) that the formulas (7.6.1) determine a continuous real function $h^{\rightarrow}(g)$ in $\mathrm{C}(M)$.

The last statement is easy to check.

It should be remarked that $h^{\rightarrow}$ is a right (Galois) adjoint of $h^{\leftarrow}$, that is,

$$
h^{\leftarrow}(f) \leqslant g \Longleftrightarrow f \leqslant h^{\rightarrow}(g)
$$

for every $f \in \operatorname{LSC}(M)$ and $g \in \operatorname{NLSC}(L)$. When we restrict the class of real functions on the left to $\mathrm{C}(M)$ this Galois connection yields an order isomorphism:

Theorem 7.7. Let $h: L \rightarrow M$ be a proper essential embedding with $M$ an extremally disconnected frame. The map

$$
h^{\rightarrow}: \operatorname{NLSC}(L) \rightarrow \mathrm{C}(M)
$$

is an order isomorphism, with inverse

$$
h^{\leftarrow}: \mathrm{C}(M) \rightarrow \operatorname{NLSC}(L) .
$$

Proof. As seen above, both $h^{\rightarrow}$ and $h^{\leftarrow}$ are well-defined order-preserving maps. It remains to check that $h^{\rightarrow}$ is a bijection with inverse $h^{\leftarrow}$.

If $f \in \mathrm{C}(M)$ then, by Proposition 7.5, $h^{\leftarrow}(f) \in \operatorname{NLSC}(L)$ and by Proposition 7.6, $h^{\rightarrow}\left(h^{\leftarrow}(f)\right) \in \mathrm{C}(M)$. By (7.5.1) we obtain that $h^{\leftarrow}(f)(r,-)=$ $\mathfrak{c}\left(h_{*}\left(f_{r}\right)\right)$ for each $r \in \mathbb{Q}$ and so $\left.h^{\leftarrow}(f)_{r}=h_{*}\left(f_{r}\right)\right)$. Applying (7.6.1), (7.5.1), Lemma 7.2(2) and Remark 6.1(4) we obtain for each $p \in \mathbb{Q}$

$$
\begin{aligned}
h^{\rightarrow}\left(h^{\leftarrow}(f)\right)(p,-) & =\bigvee_{r>p} \mathfrak{c}\left(h\left(h^{\leftarrow}(f)_{r}\right)^{* *}\right)=\bigvee_{r>p} \mathfrak{c}\left(h\left(h_{*}\left(f_{r}\right)\right)^{* *}\right) \\
& =\bigvee_{r>p} \mathfrak{c}\left(\left(f_{r}\right)^{* *}\right)=\mathfrak{c}\left(\bigvee_{r>p}\left(f_{r}\right)^{* *}\right)=\mathfrak{c}\left(f_{p}\right)=f(p,-) .
\end{aligned}
$$

Hence $h^{\rightarrow}\left(h^{\leftarrow}(f)\right)=f$.

On the other hand, starting with a $g \in \operatorname{NLSC}(L)$, then $h \rightarrow(g) \in \mathrm{C}(M)$ and $h^{\leftarrow}\left(h^{\rightarrow}(g)\right) \in \operatorname{NLSC}(L)$. By (7.6.1) we have $h^{\rightarrow}(g)(p,-)=\mathfrak{c}\left(\bigvee_{r>p} h\left(g_{r}\right)^{* *}\right)$ for every $p \in \mathbb{Q}$ and so $h^{\rightarrow}(g)_{p}=\bigvee_{r>p} h\left(g_{r}\right)^{* *}$. On the other hand, by (7.5.1), Lemma 7.2(1) and Remark 6.1(4), and since $h_{*}$ preserves directed joins, it follows that

$$
\begin{aligned}
h^{\leftarrow}\left(h^{\rightarrow}(g)\right)(p,-) & =\mathfrak{c}\left(h_{*}\left(h^{\rightarrow}(g)_{p}\right)\right)=\mathfrak{c}\left(h_{*}\left(\bigvee_{r>p} h\left(g_{r}\right)^{* *}\right)\right) \\
& =\bigvee_{r>p} \mathfrak{c}\left(h_{*}\left(h\left(g_{r}\right)^{* *}\right)\right)=\bigvee_{r>p} \mathfrak{c}\left(h_{*}\left(h\left(g_{r}\right)\right)^{* *}\right) \\
& =\bigvee_{r>p} \mathfrak{c}\left(\left(g_{r}\right)^{* *}\right)=\mathfrak{c}\left(\bigvee_{r>p}\left(g_{r}\right)^{* *}\right)=\mathfrak{c}\left(g_{p}\right)=g(p,-)
\end{aligned}
$$


for every $p \in \mathbb{Q}$. Hence $h^{\leftarrow}\left(h^{\rightarrow}(g)\right)=g$.

Corollary 7.8. Let $L$ be a completely regular frame and let $\gamma_{L}: L \rightarrow \mathfrak{G}(L)$

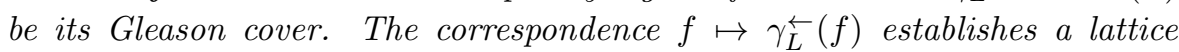
isomorphism between $\mathrm{C}(\mathfrak{G}(L))$ and $\operatorname{NLSC}(L)$.

It now follows immediately from Corollaries 5.2 and 7.8 that for weak cbframes $L$ the Dedekind completion of $\mathrm{C}(L)$ is indeed isomorphic to $\mathrm{C}(M)$ for some frame $M$. More specifically:

Corollary 7.9. Let $L$ be a completely regular, weak cb-frame. The Dedekind completion of $\mathrm{C}(L)$ is isomorphic to $\mathrm{C}(\mathfrak{G}(L))$.

This is the pointfree counterpart of the classical result, originally due to Mack and Johnson [28], that for any completely regular, weak cb-space $X$ and its minimal projective extension $Y$, the Dedekind completion of $\mathrm{C}(X)$ is isomorphic to $\mathrm{C}(Y)$.

Remark 7.10. The above corollary shows in particular that the Dedekind completion of $\mathrm{C}(L)$ is a lattice-ordered ring whenever $L$ is a completely regular weak cb-frame. Besides, one may wonder if this also holds in the more general case of not necessarily weak cb-frames, namely, if the algebraic operations of $\mathrm{C}(L)$ can be extended to the completion in such a way that the latter becomes a lattice-ordered ring. We point out that this question was already answered in the affirmative in [29, Remark 3.11]. Notice that there is a misprint in that Remark: it should say that the operations on $\mathrm{C}(L)$ can be easily extended to $\mathrm{C}^{\mathrm{X}}(L)$ (not $\left.\mathrm{IC}(L)\right)$. We take this occasion to correct a further inaccuracy in [29], on the misuse of the word "ring" in the first sentence of its abstract: indeed, the class $\operatorname{IC}(L)$ of all partial real functions on a frame is not in general an ordered ring.

\section{A third representation: Hausdorff continuous functions}

Dropping the relation (r2) from the definition of the frame of reals (in 2.2) yields the frame $\mathfrak{L}(\mathbb{I R})$ of partial real numbers [29]. Frame homomorphisms $\mathfrak{L}(\mathbb{I R}) \rightarrow L$ are called continuous partial real functions [29] on $L$.

The set

$$
\mathrm{IC}(L)
$$

of continuous partial real functions on $L$ is partially ordered by $f \leqslant g$ iff

$$
f(p,-) \leqslant g(p,-) \quad \text { and } \quad g(-, q) \leqslant f(-, q)
$$

for every $p, q \in \mathbb{Q}$.

We call any $f$ in

$$
\operatorname{IF}(L)=\operatorname{IC}(\mathcal{S}(L))=\operatorname{Frm}(\mathfrak{L}(\mathbb{R}), \mathcal{S}(L))
$$

an arbitrary partial real function on $L$. As for total real functions, we say that $f$ is lower (resp. upper) semicontinuous if $f(p,-) \in \mathfrak{c}(L)$ (resp. $f(-, p) \in \mathfrak{c}(L))$ 
for every $p \in \mathbb{Q}$. Further, $\operatorname{IC}(L)$ can be seen as the subclass of $\operatorname{IF}(L)$ of all lower and upper semicontinuous real functions.

Remark 8.1. The obvious order embedding $\iota: \mathfrak{L}(\mathbb{I} \mathbb{R}) \rightarrow \mathfrak{L}(\mathbb{R})$ defined by $(p, q) \mapsto(p, q)$ induces an embedding $I: \mathrm{F}(L) \rightarrow \mathrm{IF}(L)$ (given by $f \mapsto f \cdot \iota)$. So we may look to $\mathrm{F}(L)$ as a subset of $\operatorname{IF}(L)$, specifically as the subset of partial real functions such that

$$
f(p,-) \vee f(-, q)=1 \quad \text { for every } p<q \text { in } \mathbb{Q} .
$$

Similarly, we can embed $\mathrm{C}(L), \operatorname{LSC}(L)$ and $\operatorname{USC}(L)$ in $\operatorname{IF}(L)$ :

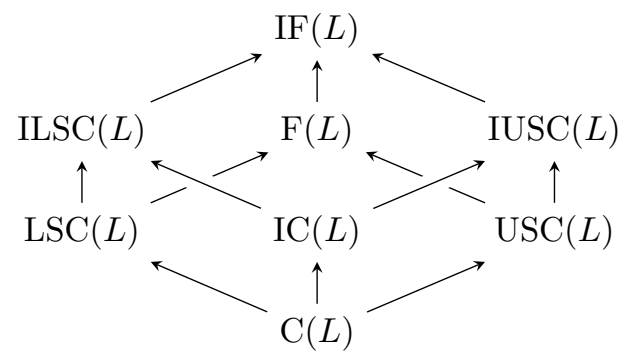

As for real functions (recall Definition 3.1), a partial real function $f \in \operatorname{IF}(L)$ is

(1) bounded if there exist $p<q$ in $\mathbb{Q}$ such that $f(p,-)=1=f(-, q)$;

(2) continuously bounded if there exist $h_{1}, h_{2} \in \mathrm{C}(L)$ such that $h_{1} \leqslant f \leqslant h_{2}$;

(3) locally bounded if

$$
\bigvee_{r \in \mathbb{Q}} \overline{f(r,-)}=1=\bigvee_{r \in \mathbb{Q}} \overline{f(-, r)} .
$$

We denote the corresponding collections of real functions by $\operatorname{IF}^{*}(L), \operatorname{IF}^{c b}(L)$ and $\mathrm{IF}^{l b}(L)$ respectively.

Remark 8.2. Obviously, bounded partial real functions and continuous functions are continuously bounded and any continuously bounded partial real function is locally bounded. Thus

$$
\operatorname{IF}^{*}(L) \cup \operatorname{IC}(L) \subseteq \operatorname{IF}^{c b}(L) \subseteq \operatorname{IF}^{l b}(L) .
$$

In order to extend the lower and upper regularizations of a real function (3.2.1-3.2.2) to partial real functions we need the following result.

Lemma 8.3. Let $f \in \operatorname{IF}^{l b}(L)$. Then $\sigma: \mathbb{Q} \rightarrow \mathcal{S}(L)$, defined by $\sigma(r)=\overline{f(r,-)}$, is a scale in $\mathcal{S}(L)$.

Proof. Since $\sigma$ is clearly antitone and each $\sigma(r)$ is complemented, it follows from Remark 2.5 that it is an extended scale. On the other hand, since $f$ is locally bounded and $0=f(r,-) \wedge f(-, r) \geq \overline{f(r,-)} \wedge \overline{f(-, r)}$ for every $r \in \mathbb{Q}$ we have

$$
\bigvee_{r \in \mathbb{Q}} \sigma(r)=\bigvee_{r \in \mathbb{Q}} \overline{f(r,-)}=1 \text { and } \bigvee_{r \in \mathbb{Q}} \sigma(r)^{*}=\bigvee_{r \in \mathbb{Q}}(\overline{f(r,-)})^{*} \geq \bigvee_{q \in \mathbb{Q}} \overline{f(-, r)}=1
$$


and thus $\sigma$ is a scale in $\mathcal{S}(L)$.

Note also that Remark 2.4.1(1) has its counterpart in $\operatorname{IF}(L)$ and there is a dual order-isomorphism $-(\cdot): \operatorname{ILSC}(L) \rightarrow \operatorname{IUSC}(L)$ defined by

$$
(-f)(-, r)=f(-r,-) \quad \text { for all } r \in \mathbb{Q} .
$$

When restricted to $\operatorname{ILSC}^{l b}(L)$ it becomes a dual isomorphism from $\operatorname{ILSC}(L)^{l b}$ onto $\operatorname{IUSC}(L)^{l b}$. With the help of the lemma, it is now a straightforward exercise to check that the lower and upper regularizations defined in Section 3 are immediately extendable to any $f \in \operatorname{IF}^{l b}(L)$ yielding operators

$$
(\cdot)^{\circ}: \operatorname{IF}^{l b}(L) \rightarrow \operatorname{LSC}(L) \text { and }(\cdot)^{-}: \operatorname{IF}^{l b}(L) \rightarrow \operatorname{USC}(L)
$$

with properties similar to the ones in Proposition 3.3 and Corollary 3.4. In particular:

Proposition 8.4. The following properties hold for any $f, g \in \mathrm{IF}^{l b}(L)$ :

(1) $f^{\circ} \leqslant f \leqslant f^{-}$.

(2) $f^{\circ \circ}=f^{\circ}$ and $f^{--}=f^{-}$.

(3) $f^{\circ} \leqslant g^{\circ}$ and $f^{-} \leqslant g^{-}$whenever $f \leqslant g$.

(4) $f^{\circ-\circ-}=f^{\circ-}$ and $f^{-\circ-\circ}=f^{-\circ}$.

Definition 8.5. An $f \in \operatorname{IF}^{l b}(L)$ is Hausdorff continuous if $f \in \operatorname{IC}(L)$, i.e., $f(p,-), f(-, q) \in \mathfrak{c}(L)$ for every $p, q \in \mathbb{Q}, f^{\circ-}=f^{-}$and $f^{-\circ}=f^{\circ}$.

We denote by $\mathrm{H}(L)$ the collection of all Hausdorff continuous partial real functions on $L$.

Obviously, $\mathrm{C}(L) \subseteq \mathrm{H}(L) \subseteq \mathrm{IC}(L)$ since $f$ is continuous if and only if $f=$ $f^{\circ}=f^{-}$. Moreover, $f^{-} \in \operatorname{NUSC}(L)$ and $f^{\circ} \in \operatorname{NLSC}(L)$ for every $f \in \mathrm{H}(L)$.

We conclude the paper with the promised third representation for the Dedekind completion of $\mathrm{C}(L)$.

Theorem 8.6. Let $L$ be a completely regular frame. The Dedekind completion of $\mathrm{C}(L)$ is isomorphic with $\mathrm{H}^{c b}(L)=\mathrm{H}(L) \cap \mathrm{IF}^{c b}(L)$.

Proof. For each $f \in \mathrm{H}(L)$, let $\Phi(f)=f^{\circ}$. By (8.3.1), $\Phi(f) \in \operatorname{LSC}(L)$. Moreover, $\Phi(f)^{-}=f^{\circ-}=f^{-} \in \mathrm{F}(L)$ and $\Phi(f)^{-\circ}=f^{\circ-\circ}=f^{-\circ}=f^{\circ}=\Phi(f)$. Thus $\Phi(f) \in \operatorname{NLSC}(L)$.

The map $\Phi: \mathrm{H}(L) \rightarrow \mathrm{NLSC}(L)$ is order-preserving and its restriction to $\mathrm{C}(L)$ is the identity map. Hence $\Phi(f) \in \operatorname{NLSC}^{c b}(L)$ whenever $f \in \mathrm{H}^{c b}(L)$, and $\Phi_{\mid \mathrm{H}^{c b}(L)}$ is an order-preserving map from $\mathrm{H}^{c b}(L)$ into $\operatorname{NLSC}^{c b}(L)$.

Conversely, given $g \in \operatorname{NLSC}(L)$ and $p, q \in \mathbb{Q}$ define

$$
\Psi(g)(p,-)=g(p,-) \quad \text { and } \quad \Psi(g)(-, q)=g^{-}(-, q) .
$$

In order to show that $\Psi(g) \in \operatorname{IF}(L)$ we only need to prove that $\Psi(g)$ turns the defining relations $(\mathrm{r} 1)$ and $(\mathrm{r} 3)-(\mathrm{r} 6)$ into identities in $\mathcal{S}(L)$ :

(r1) For each $p \geq q$, it follows from Remarks 6.1 that

$$
\Psi(g)(p,-) \wedge \Psi(g)(-, q)=g(p,-) \wedge g^{-}(-, q) \leqslant g(p,-) \wedge g(-, q)=0 .
$$


(r3)-(r6) follow since $g \in \operatorname{NLSC}(L)$ and $g^{-} \in \operatorname{NUSC}(L)$. Further,

$$
\begin{aligned}
& \bigvee_{r \in \mathbb{Q}} \overline{\Psi(g)(r,-)}=\bigvee_{r \in \mathbb{Q}} \overline{g(r,-)}=\bigvee_{r \in \mathbb{Q}} g(r,-)=1 \quad \text { and } \\
& \bigvee_{r \in \mathbb{Q}} \overline{\Psi(g)(-, r)}=\bigvee_{r \in \mathbb{Q}} \overline{g^{-}(-, r)}=\bigvee_{r \in \mathbb{Q}} g^{-}(-, r)=1,
\end{aligned}
$$

which ensures that $\Psi(g) \in \mathrm{IF}^{l b}(L)$. Moreover,

$$
\begin{aligned}
& \Psi(g)^{\circ}(p,-)=\bigvee_{r>p} \overline{\Psi(g)(r,-)}=\bigvee_{r>p} \overline{g(r,-)}=g(p,-) \quad \text { and } \\
& \Psi(g)^{-}(-, q)=\bigvee_{s<q} \overline{\Psi(g)(-, s)}=\bigvee_{s<q} \overline{g^{-}(-, s)}=g(-, q)
\end{aligned}
$$

for every $p, q \in \mathbb{Q}$. Hence $\Psi(g)^{\circ}=g, \Psi(g)^{-}=g^{-}, \Psi(g)^{\circ-}=g^{-}$and $\Psi(g)^{-\circ}=$ $g^{-\circ}=g$ and so $\Psi(g) \in \mathrm{H}(L)$.

It is also easy to check that $\Psi: \operatorname{NLSC}(L) \rightarrow \mathrm{H}(L)$ is order-preserving and its restriction to $\mathrm{C}(L)$ is the identity. Therefore, $\Psi(g) \in \mathrm{H}^{c b}(L)$ whenever $g \in \operatorname{NLSC}(L)$, and $\Psi_{\mid \mathrm{NLSC}^{c b}(L)}$ is an order-preserving map from $\operatorname{NLSC}^{c b}(L)$ into $\mathrm{H}^{c b}(L)$.

Finally, for each $f \in \mathrm{H}^{c b}(L), g \in \operatorname{NLSC}^{c b}(L)$ and $p, q \in \mathbb{Q}$, we have that

$$
\begin{aligned}
\Psi(\Phi(f))(p,-)= & \Phi(f)(p,-)=f^{\circ}(p,-)=\bigvee_{r>p} \overline{f(r,-)}=\bigvee_{r>p} f(r,-)=f(p,-), \\
\Psi(\Phi(f))(-, q) & =\Phi(f)^{-}(-, q)=\bigvee_{s<q} \overline{\Phi(f)(-, s)}=\bigvee_{s<q} \overline{f^{\circ}(-, s)}=f^{\circ-}(-, q) \\
& =f^{-}(-, q)=\bigvee_{s<q} \overline{f(-, s)}=\bigvee_{s<q} f(-, s)=f(-, q) \quad \text { and } \\
\Phi(\Psi(g))(p,-) & =\Psi(g)^{\circ}(p,-)=\bigvee_{r>p} \overline{\Psi(g)(r,-)}=\bigvee_{r>p} \overline{g(r,-)}=\bigvee_{r>p} g(r,-) \\
& =g(p,-),
\end{aligned}
$$

that is, $\Psi \cdot \Phi=1_{\mathrm{H}^{c b}(L)}$ and $\Phi \cdot \Psi=1_{\mathrm{NLSC}^{c b}(L)}$.

This is the pointfree version of Anguelov's characterization in [1] of the Dedekind completion of $\mathrm{C}(X)$ in a constructive form, as a set of real functions on the same space $X$.

Acknowledgments. Research supported by the Ministry of Economy and Competitiveness of Spain (through grant MTM2012-37894-C02-02), the University of the Basque Country UPV/EHU (through grants UFI11/52 and GIU12/39) and the Centre for Mathematics of the University of Coimbra (funded by the ERD Fund through the program COMPETE and by the Portuguese Government/FCT through grant PEst-C/MAT/UI0324/2011).

I. Mozo Carollo gratefully acknowledges financial assistance from a Predoctoral Fellowship of the Basque Country Government (BFI-2012-262). 


\section{References}

[1] Anguelov R.: Dedekind order completion of $C(X)$ by Hausdorff continuous real functions. Quaest. Math. 27, 153-169 (2004)

[2] Banaschewski B.: Compact regular frames and the Sikorski Theorem. Kyungpook Math. J. 28, 1-14 (1988)

[3] Banaschewski B.: The real numbers in pointfree topology. Textos de Matemática, vol. 12, Departamento de Matemática da Universidade de Coimbra (1997)

[4] Banaschewski B., Gutiérrez García J., Picado J.: Extended real functions in pointfree topology. J. Pure Appl. Algebra 216, 905-922 (2012)

[5] Banaschewski B., Hager A.W.: Essential completeness in categories of completely regular frames. Appl. Categ. Structures 21, 167-180 (2013)

[6] Banaschewski B., Hong S.S.: Completeness properties of function rings in pointfree topology. Comment. Math. Univ. Carolin. 44, 245-259 (2003)

[7] Banaschewski B., Pultr A.: Booleanization. Cahiers Topologie Géom. Différentielle Catég. 37, 41-60 (1996)

[8] Chen X.: Closed frame homomorphisms. PhD Thesis, McMaster University (1991)

[9] Dăneţ N.: The Dedekind completion of $C(X)$ : An interval-valued functions approach. Quaest. Math. 34 213-223 (2011)

[10] Dieudonné J.: Review of [14]. Math. Reviews 22, 1190-1191 (1961)

[11] Dilworth R.P.: The normal completion of the lattice of continuous functions. Trans. Amer. Math. Soc. 68, 427-438 (1950)

[12] Dube T.: An algebraic view of weaker forms of realcompactness. Algebra Universalis 55, 187-202 (2006)

[13] Dube T.: Realcompactness and certain types of subframes. Algebra Universalis 58, 181-202 (2008)

[14] Gillman L., Jerison M.: Rings of continuous functions. D. Van Nostrand (1960)

[15] Gutiérrez García J., Kubiak T.: General insertion and extension theorems for localic real functions. J. Pure Appl. Algebra 215, 1198-1204 (2011)

[16] Gutiérrez García J., Kubiak T., Picado J.: Lower and upper regularizations of frame semicontinuous real functions. Algebra Universalis 60, 169-184 (2009)

[17] Gutiérrez García J., Kubiak T., Picado J.: Pointfree forms of Dowker's and Michael's insertion theorems. J. Pure Appl. Algebra 213, 98-108 (2009)

[18] Gutiérrez García J., Kubiak T., Picado J.: Localic real functions: A general setting. J. Pure Appl. Algebra 213, 1064-1074 (2009)

[19] Gutiérrez García J., Picado J.: On the algebraic representation of semicontinuity. J. Pure Appl. Algebra 210, 299-306 (2007)

[20] Gutiérrez García J., Picado J.: Rings of real functions in pointfree topology. Topology Appl. 158, 2264-2278 (2011)

[21] Gutiérrez García J., Picado J.: On the parallel between normality and extremal disconnectedness. J. Pure Appl. Algebra 218, 784-803 (2014)

[22] Horn A.: The normal completion of a subset of a complete lattice and lattices of continuous functions. Pacific J. Math. 3, 137-152 (1953)

[23] Horne J.G.: Countable paracompactness and cb-spaces. Notices Amer. Math. Soc. 6, 629-630 (1959)

[24] Johnson D.G.: The completion of an archimedean $f$-ring. J. London Math. Soc. 40, 493-496 (1965)

[25] Johnstone P.T.: The Gleason cover of a topos, I. J. Pure Appl. Algebra 22, 171-192 (1980)

[26] Johnstone P.T.: Stone Spaces. Cambridge Studies in Advanced Mathematics, Vol. 3, Cambridge University Press (1982)

[27] Mack J.E.: On a class of countably paracompact spaces. Proc. Amer. Math. Soc. 16, 467-472 (1965)

[28] Mack J.E., Johnson D.G.: The Dedekind completion of $C(\mathcal{X})$. Pacific J. Math. 20, 231-243 (1967)

[29] Mozo Carollo I., Gutiérrez García J., Picado J.: On the Dedekind completion of function rings. Forum Math. (doi: 10.1515/forum-2012-0095, in press) 
[30] Nakano H.: Über das System aller stetigen Funktionen auf einen topologischen Raum. Proc. Imp. Acad. Tokyo 17, 308-310 (1941) (German)

[31] Picado J., Pultr A.: Frames and locales: Topology without points. Frontiers in Mathematics, vol. 28, Springer, Basel (2012)

[32] Steinberg S.A.: Lattice-ordered rings and modules. Springer, New York (2010)

[33] Stone M.H.: Boundedness properties in function lattices. Canad. J. Math. 1, 176-186 (1949)

JaVier GutiÉRRez García

Department of Mathematics, University of the Basque Country UPV/EHU, Apdo. 644, 48080 Bilbao, Spain

e-mail: javier.gutierrezgarcia@lg.ehu.es

URL: http://www.ehu.es/javiergutierrezgarcia

Imanol Mozo Carollo

Department of Mathematics, University of the Basque Country UPV/EHU, Apdo. 644, 48080 Bilbao, Spain

e-mail: imanol.mozo@ehu.es

Jorge PicAdo

CMUC, Department of Mathematics, University of Coimbra, 3001-501 Coimbra,

Portugal

e-mail: picado@mat.uc.pt

$U R L:$ http://www.mat.uc.pt/ picado 